\title{
QUEEN'S
UNIVERSITY
BELFAST
}

\section{NGTS 15b, 16b, 17b, and 18b: four hot Jupiters from the Next- Generation Transit Survey}

Tilbrook, R. H., Burleigh, M. R., Costes, J. C., Gill, S., Nielsen, L. D., Vines, J. I., Queloz, D., Hodgkin, S. T., Worters, H. L., Goad, M. R., Acton, J. S., Henderson, B. A., Armstrong, D. J., Anderson, D. R., Bayliss, D., Bouchy, F., Briegal, J. T., Bryant, E. M., Casewell, S. L., ... Alves, D. R. (2021). NGTS 15b, 16b, 17b, and 18b: four hot Jupiters from the Next-Generation Transit Survey. Monthly Notices of the Royal Astronomical Society, 504(4), 6018-6032. https://doi.org/10.1093/mnras/stab815

Published in:

Monthly Notices of the Royal Astronomical Society

Document Version:

Peer reviewed version

Queen's University Belfast - Research Portal:

Link to publication record in Queen's University Belfast Research Portal

Publisher rights

Copyright 2021 The Author(s) Published by Oxford University Press on behalf of the Royal Astronomical Society.

This work is made available online in accordance with the publisher's policies. Please refer to any applicable terms of use of the publisher.

\section{General rights}

Copyright for the publications made accessible via the Queen's University Belfast Research Portal is retained by the author(s) and / or other copyright owners and it is a condition of accessing these publications that users recognise and abide by the legal requirements associated with these rights.

Take down policy

The Research Portal is Queen's institutional repository that provides access to Queen's research output. Every effort has been made to ensure that content in the Research Portal does not infringe any person's rights, or applicable UK laws. If you discover content in the Research Portal that you believe breaches copyright or violates any law, please contact openaccess@qub.ac.uk. 


\section{NGTS 15b, 16b, 17b and 18b: four hot Jupiters from the Next Generation Transit Survey}

Rosanna H. Tilbrook, ${ }^{1 \star}$ Matthew R. Burleigh, ${ }^{1}$ Jean C. Costes, ${ }^{2}$ Samuel Gill, $, 3,4$ Louise Dyregaard Nielsen, ${ }^{5}$ José I. Vines, ${ }^{6}$ Didier Queloz, ${ }^{7}$ Simon T. Hodgkin, ${ }^{8}$ Hannah L. Worters, ${ }^{9}$ Michael R. Goad, ${ }^{1}$ Jack S. Acton, ${ }^{1}$ Beth A. Henderson, ${ }^{1}$

David J. Armstrong, ${ }^{3,4}$ David R. Anderson, ${ }^{3,4}$ Daniel Bayliss, ${ }^{3,4}$ François Bouchy, ${ }^{5}$ Joshua T. Briegal, ${ }^{7}$ Edward M. Bryant, ${ }^{3,4}$ Sarah L. Casewell, ${ }^{1}$ Alexander Chaushev, ${ }^{10}$ Benjamin F. Cooke, ${ }^{3,4}$ Philipp Eigmüller, ${ }^{11}$ Edward Gillen, ${ }^{12,7 \dagger}$ Maximilian N. Günther, ${ }^{13}$ Aleisha Hogan, ${ }^{1}$ James S. Jenkins, ${ }^{6,14}$ Monika Lendl, ${ }^{5}$ James McCormac, ${ }^{3,4}$ Maximiliano Moyano, ${ }^{15}$ Liam Raynard, ${ }^{1}$ Alexis M. S. Smith, ${ }^{11}$ Stéphane Udry, ${ }^{5}$ Christopher A. Watson, ${ }^{2}$ Richard G. West, ${ }^{3,4}$ Peter J. Wheatley, ${ }^{3,4}$ Hannes Breytenbach, ${ }^{9}, 16$ Ramotholo R. Sefako, ${ }^{9}$ Jessymol K. Thomas, ${ }^{9}$ Douglas R. Alves $^{6}$

\footnotetext{
${ }^{1}$ School of Physics and Astronomy, University of Leicester, LE1 7RH, UK

${ }^{2}$ Astrophysics Research Centre, School of Mathematics and Physics, Queen's University Belfast, BT7 1NN Belfast, UK

${ }^{3}$ Centre for Exoplanets and Habitability, University of Warwick, Gibbet Hill Road, Coventry CV4 7AL, UK

${ }^{4}$ Dept. of Physics, University of Warwick, Gibbet Hill Road, Coventry CV4 7AL, UK

${ }^{5}$ Observatoire de Genève, Université de Genève, 51 Ch. des Maillettes, 1290 Sauverny, Switzerland

${ }^{6}$ Departamento de Astronomia, Universidad de Chile, Casilla 36-D, Santiago, Chile

${ }^{7}$ Astrophysics Group, Cavendish Laboratory, J.J. Thomson Avenue, Cambridge CB3 OHE, UK

${ }^{8}$ Institute of Astronomy, University of Cambridge, Madingley Rise, Cambridge CB3 OHA, UK

${ }^{9}$ South African Astronomical Observatory, P.O Box 9, Observatory 7935, Cape Town, South Africa

${ }^{10}$ Center for Astronomy and Astrophysics, TU Berlin, Hardenbergstr. 36, D-10623 Berlin, Germany

${ }^{11}$ Institute of Planetary Research, German Aerospace Center, Rutherfordstrasse 2, 12489 Berlin, Germany

${ }^{12}$ Astronomy Unit, Queen Mary University of London, Mile End Road, London E1 4NS, UK

${ }^{13}$ Department of Physics, and Kavli Institute for Astrophysics and Space Research, Massachusetts Institute of Technology,

Cambridge, MA 02139, USA

${ }^{14}$ Centro de Astrofísica y Tecnologías Afines (CATA), Casilla 36-D, Santiago, Chile.

${ }^{15}$ Instituto de Astronomía, Universidad Católica del Norte, Angamos 0610, 1270709, Antofagasta, Chile

${ }^{16}$ Department of Astronomy, University of Cape Town, Rondebosch 7700, Cape Town, South Africa
}

Accepted XXX. Received YYY; in original form ZZZ

\begin{abstract}
We report the discovery of four new hot Jupiters with the Next Generation Transit Survey (NGTS). NGTS-15b, NGTS-16b, NGTS-17b, and NGTS-18b are short-period $(P<5 \mathrm{~d})$ planets orbiting G-type main sequence stars, with radii and masses between $1.10-1.30 R_{J}$ and $0.41-0.76 M_{J}$. By considering the host star luminosities and the planets' small orbital separations $(0.039-0.052 \mathrm{AU})$, we find that all four hot Jupiters are highly irradiated and therefore occupy a region of parameter space in which planetary inflation mechanisms become effective. Comparison with statistical studies and a consideration of the planets' high incident fluxes reveals that NGTS-16b, NGTS-17b, and NGTS-18b are indeed likely inflated, although some disparities arise upon analysis with current Bayesian inflationary models. However, the underlying relationships which govern radius inflation remain poorly understood. We postulate that the inclusion of additional hyperparameters to describe latent factors such as heavy element fraction, as well as the addition of an updated catalogue of hot Jupiters, would refine inflationary models, thus furthering our understanding of the physical processes which give rise to inflated planets.
\end{abstract}

Key words: planetary systems - planets and satellites: detection - planets and satellites: gaseous planets 


\section{Rosanna H. Tilbrook et al.}

\section{INTRODUCTION}

The field of exoplanet discovery has uncovered a cosmic zoo of planetary types which extends far beyond those of our Solar System. As some of the first exoplanets ever detected, a particularly immediate revelation was the existence of Jupiter-sized planets on extremely short orbits $(P<10 \mathrm{~d})($ e.g. Mayor \& Queloz 1995; Charbonneau et al. 2000; Henry et al. 2000). In spite of their apparent rarity, comprising only $<1 \%$ of systems (Mayor et al. 2011; Wright et al. 2012; Fressin et al. 2013; Hsu et al. 2019), these 'hot Jupiters' are some of the most easily detectable exoplanets, as their large radii produce transits that are well above typical telescope noise limits, and their large masses and short orbital periods yield large radial velocity signals.

A distinctive yet poorly understood feature of the hot Jupiter population is the observation that many of these planets have radii that are larger than expected from theoretical models (e.g. Guillot \& Showman 2002; Anderson et al. 2011; Hartman et al. 2012; Espinoza et al. 2016; Raynard et al. 2018). Whilst this feature appears to correlate with incident flux (Laughlin et al. 2011; Weiss et al. 2013; Thorngren \& Fortney 2018) this alone does not provide a sufficient explanation for inflation, and thus the underlying driving mechanisms continue to be debated (see, e.g., Spiegel \& Burrows 2013, for a comprehensive review). Indeed, evolutionary models that incorporate stellar irradiation and heavy metals can explain the unexpected radii of some hot Jupiters (Fortney et al. 2007; Baraffe et al. 2008), but are yet to adequately describe the high-irradiation regime.

Efforts to characterise the relationship between inflation and incident flux have nevertheless provided valuable insight into the topic. In particular, the physical processes that cause inflation are believed to become effective in planets that are irradiated at fluxes in excess of $\sim 2 \times 10^{5} \mathrm{Wm}^{-2}$ (Demory \& Seager 2011; Miller \& Fortney 2011). Furthermore, a recent study by Sestovic et al. (2018) suggests that above an incident flux of $\sim 1.6 \times 10^{6} \mathrm{Wm}^{-2}$, all hot Jupiters in the mass range $0.37-0.98 M_{J}$ appear inflated. For statistical studies such as this, increasing the sample of well-characterised hot Jupiters is crucial, and allows us to further constrain and paramaterise inflation mechanisms, which remain poorly understood.

In this paper we report the discovery of four new hot Jupiter planets from the Next Generation Transit Survey (NGTS), three of which appear inflated. In $\$ 2$, we describe the discovery photometry from NGTS and the subsequent photometric and radial velocity follow-up observations. In $\S 3$, we present our analysis of this data, including the determination of both the stellar and planetary parameters from spectral analysis, SED fitting, and global modelling. An investigation into the inflation of each planet is covered in $\S 4$, with the subsequent results being discussed in $\$ 5$. Finally, our conclusions are laid out in $\$ 6$. NGTS-15b, NGTS-16b, NGTS-17b and NGTS-18b bring the total planet count from NGTS to 17 (note NGTS-7Ab is a brown dwarf).

\section{OBSERVATIONS}

\subsection{NGTS Discovery Photometry}

NGTS-15 to NGTS-18 were initially identified as transiting exoplanet candidate systems from photometry from the Next Genera-

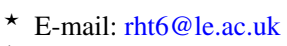

Winton Fellow

* Juan Carlos Torres Fellow
}

tion Transit Survey (NGTS; Wheatley et al. 2018), a ground-based wide-field exoplanet survey. Based at ESO's Paranal observatory in Chile, NGTS consists of an array of 12 independently mounted $20-\mathrm{cm}$ Newtonian telescopes, each equipped with a $2 \mathrm{~K} \times 2 \mathrm{~K}$ deepdepleted Andor IKon-L CCD camera. The custom NGTS 520$890 \mathrm{~nm}$ filter optimises the survey for studies of K and M dwarf stars which, due to their small radii, provide the best opportunity for discovering small transiting planets.

NGTS-15, NGTS-16, and NGTS-17 were observed during the 2017 observing campaign for 160, 137, and 160 nights, respectively, between 2017 August 16 and 2018 March 18. NGTS-18 was observed the following season for 165 nights over the period of 2017 December 10 to 2018 August 7. Over 188,000 images were collected for each object using a single NGTS telescope with $10 \mathrm{~s}$ exposures (see Table 2 for details).

The data were reduced and aperture photometry performed via the CASUTools package ${ }^{1}$, before being detrended using an optimised version of the SysRem algorithm (Tamuz et al. 2005) which has been adapted for the NGTS pipeline. The data was then searched for transit-like events by ORION (Wheatley et al. 2018), a custom implementation of the BLS fitting algorithm (Kovács et al. 2002). ORION identified eight partial and three full transits for NGTS-15; four partial and four full transits for NGTS-16; 15 partial transits for NGTS-17; and 16 partial and four full transits for NGTS-18. Lightcurves of the NGTS detections, phase-folded on the best-fitting period, are shown in Figure 1. The initial fits to the NGTS data provided by ORION revealed that the depths, widths and shapes of the transits for each object were compatible with transiting hot Jupiter planets. In addition, a convolutional neural network applied to the NGTS data found that the probabilities of each lightcurve containing a transiting exoplanet were all greater than 0.95 , consistent with previous confirmed NGTS planet discoveries (Chaushev et al. 2019).

In order to rule out the possibility that any of our detected companions were stellar rather than planetary, we performed additional checks on the NGTS data. The phase-folded lightcurves for each object were searched for any evidence of a secondary eclipse around phase 0.5 which would indicate the presence of a second star. Furthermore, we compared the transit depth of consecutive odd and even transits to check for a depth difference consistent with an eclipsing binary star system that had been mis-folded on half the true period. For all four targets, we find no indication in the NGTS photometry to suggest that the companions were not planets.

\subsection{Additional Photometry}

In order to confirm that each stellar companion was indeed a planet, and to constrain the transit parameters, we obtained additional photometry with the $1.0 \mathrm{~m}$ and Lesedi telescopes at the South African Astronomical Observatory (SAAO). For three of the candidates, we were also able to use data from the Transiting Exoplanet Survey Satellite (Ricker et al. 2014). The details of this additional photometry are outlined below.

\subsubsection{TESS}

TESS is a space-based NASA survey telescope that searches for transiting planets around bright stars (Ricker et al. 2014). It has a

\footnotetext{
1 http://casu.ast.cam.ac.uk/surveys-projects/ software-release
} 
Table 1. NGTS photometry for each object. The full tables are available in a machine-readable format from the online journal. A portion is shown here for guidance.

(a) NGTS photometry for NGTS-15.

\begin{tabular}{ccc}
\hline $\begin{array}{c}\text { Time } \\
\text { (BJD-2450000) }\end{array}$ & $\begin{array}{c}\text { Flux } \\
\text { (normalised) }\end{array}$ & $\begin{array}{c}\text { Flux } \\
\text { error }\end{array}$ \\
\hline 7982.8536 & 0.973 & 0.031 \\
7982.8538 & 0.973 & 0.031 \\
7982.8539 & 1.001 & 0.031 \\
7982.8541 & 1.008 & 0.031 \\
7982.8542 & 1.023 & 0.031 \\
$\ldots$ & $\ldots$ & $\ldots$ \\
8195.5583 & 1.012 & 0.030 \\
8195.5584 & 1.039 & 0.030 \\
8195.5586 & 0.974 & 0.030 \\
8195.5587 & 0.959 & 0.030 \\
8195.5589 & 1.025 & 0.030 \\
\hline
\end{tabular}

(c) NGTS photometry for NGTS-17.

\begin{tabular}{ccc}
\hline $\begin{array}{c}\text { Time } \\
\text { (BJD-2450000) }\end{array}$ & $\begin{array}{c}\text { Flux } \\
\text { (normalised) }\end{array}$ & $\begin{array}{c}\text { Flux } \\
\text { error }\end{array}$ \\
\hline 7982.8537 & 1.001 & 0.025 \\
7982.8539 & 1.021 & 0.025 \\
7982.8540 & 1.014 & 0.025 \\
7982.8542 & 1.002 & 0.025 \\
7982.8543 & 1.023 & 0.025 \\
$\ldots$ & $\ldots$ & $\ldots$ \\
8195.5583 & 0.927 & 0.025 \\
8195.5584 & 0.997 & 0.025 \\
8195.5586 & 1.032 & 0.025 \\
8195.5587 & 0.984 & 0.025 \\
8195.5589 & 1.025 & 0.025 \\
\hline
\end{tabular}

wide field of view, with four $24 \times 24^{\circ}$ cameras, each equipped with four $2 \mathrm{k} \times 2 \mathrm{k}$ CCDs. Its typical observing baseline of 27 days makes it well-suited to detecting short-period transiting exoplanets.

We searched for our candidates in the TESS full frame images (FFIs) using the TESSCut ${ }^{2}$ tool and found that NGTS-16 and NGTS-17 were observed in TESS Sectors 4 and 5, respectively, each for 27 consecutive nights between 2018 October 19 and 2018 December 11. In addition, NGTS-18 was observed between 2019 March 26 and 2019 April 22 in TESS Sector 10. NGTS-15 falls on a CCD in TESS Sector 5, but unfortunately into the overscan region of the camera rather than the science pixels, and therefore there is no TESS FFI observation for this star. A summary of this information can be found in Table 2 .

Each star in the TESS FFIs was observed at 30 minute cadence, and for each of our candidates we used an automatically determined optimal aperture to exclude neighbouring stars. These aperture sizes ranged from three to eight pixels. However, the large pixel scale of TESS (21") meant that there was still some slight contamination of the FFI lightcurves, which we account for in our analysis (see Section 3.2).

For each candidate, we found that the best BLS period from TESS was consistent with the ORION value for the NGTS photome-

\footnotetext{
2 https://mast.stsci.edu/tesscut/
}

(b) NGTS photometry for NGTS-16.

\begin{tabular}{ccc}
\hline $\begin{array}{c}\text { Time } \\
\text { (BJD-2450000) }\end{array}$ & $\begin{array}{c}\text { Flux } \\
\text { (normalised) }\end{array}$ & $\begin{array}{c}\text { Flux } \\
\text { error }\end{array}$ \\
\hline 7981.7857 & 1.010 & 0.031 \\
7981.7860 & 0.968 & 0.031 \\
7981.7862 & 0.985 & 0.031 \\
7981.7863 & 1.007 & 0.031 \\
7981.7865 & 1.008 & 0.031 \\
$\ldots$ & $\ldots$ & $\ldots$ \\
8191.5306 & 1.020 & 0.027 \\
8191.5307 & 0.969 & 0.027 \\
8191.5308 & 0.993 & 0.027 \\
8191.5310 & 0.994 & 0.027 \\
8191.5311 & 0.991 & 0.027 \\
\hline
\end{tabular}

(d) NGTS photometry for NGTS-18.

\begin{tabular}{ccc}
\hline $\begin{array}{c}\text { Time } \\
\text { (BJD-2450000) }\end{array}$ & $\begin{array}{c}\text { Flux } \\
\text { (normalised) }\end{array}$ & $\begin{array}{c}\text { Flux } \\
\text { error }\end{array}$ \\
\hline 8097.8232 & 1.016 & 0.032 \\
8097.8233 & 0.961 & 0.032 \\
8097.8235 & 0.977 & 0.032 \\
8097.8236 & 1.007 & 0.032 \\
8097.8238 & 1.005 & 0.032 \\
$\ldots$ & $\ldots$ & $\ldots$ \\
8334.5046 & 1.000 & 0.033 \\
8334.5047 & 0.991 & 0.033 \\
8334.5049 & 1.010 & 0.033 \\
8334.5050 & 1.026 & 0.033 \\
8334.5052 & 0.983 & 0.033 \\
\hline
\end{tabular}

try. TESS detected three full and two partial transits for NGTS-16, eight full partial transits for NGTS-17, and seven full transits for NGTS-18. Phase-folded lightcurves of the TESS data can be found in Figure 1.

\subsubsection{SAAO}

Between 2018 November and 2019 December, we took at least one set of follow-up photometry for each target using the $1.0 \mathrm{~m}$ telescope at SAAO. The telescope was equipped with the full-frame transfer CCD Sutherland High-speed Optical Camera (SHOC), "SHOC' $n$ 'awe", and all observations were taken using the $I$ band filter. The $2.85^{\prime} \times 2.85^{\prime}$ field of view of SHOC'n'awe on the $1.0 \mathrm{~m}$ telescope allowed us to simultaneously observe at least one comparison star of similar brightness for each object.

For NGTS-18, we also had the opportunity to obtain two lightcurves using the newly commissioned $1.0 \mathrm{~m}$ Lesedi telescope at SAAO, which was equipped with the "SHOC'n'disbelief" optical camera. The instrument's slightly wider field of view $\left(5.7^{\prime} \times 5.7^{\prime}\right)$ allowed for an increased selection of comparison stars.

Two lightcurves of NGTS-15 were obtained using the $1.0 \mathrm{~m}$ telescope on the nights of 2018 November 21 and 2019 December 6 with exposure times of $60 \mathrm{~s}$ and 30s, respectively. We obtained 285 images for the 2018 data, and truncated the 2019 data from 543 
Table 2. Summary of photometric observations.

\begin{tabular}{|c|c|c|c|c|c|c|}
\hline Target & Instrument & Night(s) observed & $\mathrm{N}_{\text {images }}$ & Exptime (s) & Filter & Comments \\
\hline \multirow{3}{*}{ NGTS-15 } & NGTS & $16 / 08 / 17-18 / 03 / 18$ & 196732 & 10 & NGTS & \\
\hline & SAAO & $21 / 11 / 18$ & 285 & 60 & I & \\
\hline & SAAO & $06 / 12 / 19$ & 276 & 30 & I & \\
\hline \multirow{4}{*}{ NGTS-16 } & NGTS & $16 / 08 / 17-18 / 03 / 18$ & 188417 & 10 & NGTS & \multirow{4}{*}{ Sector 4} \\
\hline & TESS & $19 / 10 / 18-14 / 11 / 18$ & 831 & $1800^{*}$ & TESS & \\
\hline & SAAO $1.0 \mathrm{~m}$ & $21 / 12 / 18$ & 200 & 60 & I & \\
\hline & SAAO $1.0 \mathrm{~m}$ & $05 / 12 / 19$ & 540 & 30 & I & \\
\hline \multirow{3}{*}{ NGTS-17 } & NGTS & $16 / 08 / 17-18 / 03 / 18$ & 196732 & 10 & NGTS & \multirow{3}{*}{ Sector 5} \\
\hline & TESS & $15 / 11 / 18-11 / 12 / 18$ & 1093 & $1800 *$ & TESS & \\
\hline & SAAO $1.0 \mathrm{~m}$ & 05/02/19 & 1104 & 15 & I & \\
\hline \multirow{5}{*}{ NGTS-18 } & NGTS & $10 / 12 / 17-07 / 08 / 18$ & 243515 & 10 & NGTS & \multirow{5}{*}{ Sector 10} \\
\hline & SAAO $1.0 \mathrm{~m}$ & $21 / 03 / 19$ & 598 & 20 & I & \\
\hline & TESS & $26 / 03 / 19-22 / 04 / 19$ & 1017 & $1800^{*}$ & TESS & \\
\hline & Lesedi & $24 / 06 / 2020$ & 426 & 15 & I & \\
\hline & Lesedi & $30 / 06 / 2020$ & 550 & 15 & $\mathrm{~V}$ & \\
\hline
\end{tabular}

The NGTS filter has a bandpass from $520-890 \mathrm{~nm}$; the TESS bandpass spans 600-1000nm.

*TESS cameras have an exposure time of $2 \mathrm{~s}$ but are stacked to $30 \mathrm{~min}$ cadence.

to 276 frames due to extremely poor conditions towards the end of the night. Similarly, additional photometry was also obtained using the same telescope on the night of 2020 February 3, but a combination of poor atmospheric conditions (with seeing reaching $6.5^{\prime \prime}$ ) and load shedding (scheduled electrical power shutdowns) at SAAO caused data gaps and fluctuations in the lightcurve on the order of the size of the transit signal, and so we omit this data from our analysis.

Load shedding also tainted the observations for NGTS-18 in 2019 March, resulting in a noticeable in-transit data gap of about 20 minutes in the final lightcurve. Despite being able to identify transit egress in this data, we omitted it from our final modelling process, as the data quality was too poor to obtain a reliable fit. However, we include the lightcurve in Figure 1d. We instead utilise follow up photometry from the Lesedi telescope, taken on the nights of 2020 June 24 and 30 in the $I$ and $V$ bands, respectively. The $I$ band data consists of $426 \times 15 \mathrm{~s}$ exposures; although it also includes a data gap of about $14 \mathrm{~min}$ long, this time due to an auto guider failure, the in-transit data is stable, and we thus include it in the modelling. Fortunately, the final dataset in $V$ band was taken continuously over $2.29 \mathrm{hr}$, and consists of $550 \times 15 \mathrm{~s}$ exposures.

NGTS-16 and NGTS-17 were both observed using only the $1.0 \mathrm{~m}$ telescope at SAAO. For NGTS-16, we obtained two follow-up lightcurves on the night of 2018 December $21(200 \times 60$ s exposures $)$ and the night of 2019 December $5(540 \times 30$ s exposures $)$, whilst NGTS-17 was observed once on the night of 2019 Febuary 5 (1104 $\times 15 \mathrm{~s}$ exposures).

A full summary of the photometric observations for each object is detailed in Table 2 .

Each lightcurve was bias and flat-field corrected using the local PYTHON-based SAAO SHOC pipeline, which uses IRAF photometry tasks (PYRAF) and facilitates the extraction of raw and differential lightcurves. We used the Starlink package ачторнотом to perform aperture photometry on both our target and comparison stars, and chose apertures that gave the maximum signal-to-noise ratio. Background apertures were adjusted to account for changes in apparent star size over the night as the atmospheric conditions varied. Finally, the measured fluxes of the comparison stars for each object were used for differential photometry of our targets.

The SAAO lightcurves of each candidate are shown in Figure 1. For NGTS-15 and NGTS-16, which each have two SAAO detections from the $1.0 \mathrm{~m}$ telescope, the data has been phase-folded on the bestfitting period.

We detected a clear egress for NGTS-15 in 2018 November, as well as an ingress in 2019 December. Although seeing reached 6.4 " near the beginning of the 2018 observations, resulting in large in-transit scatter for this lightcurve, our fitting procedure reveals that the transit depth is consistent with the NGTS data to within errors. Additionally, we note that these errors are likely underestimated for the weather-affected parts of the SAAO lightcurve.

Analysis of the 2018 December data for NGTS-16 revealed the majority of a transit, just missing ingress, whilst the 2019 December data is primarily out-of-transit with a few data points in egress. As with the 2018 data for NGTS-15, the 2018 data for NGTS-16 was affected by varying atmospheric conditions, with the full moon and high cloud causing in-transit scatter. However, again the transit depths between telescopes are in agreement.

Similar the 2018 lightcurve for NGTS-16, we detected close to a full transit for NGTS-17, again just missing ingress. Finally, the data for NGTS-18 from the Lesedi telescope contained an egress on the night of 2020 June 24 and a full transit, including some points in egress, from the night of 2020 June 30.

For all four objects, the transits observed with SAAO were consistent with planetary companions. Subsequently each were flagged for spectroscopic follow-up to enable mass determination.

\subsection{Spectroscopic Follow-Up}

In order to constrain the masses of the planetary companions, we obtained multi-epoch spectroscopy for radial velocity measurements. Three different fibre-fed échelle spectrographs were used, all located at the La Silla Observatory in Chile. The HARPS spectrograph (Mayor et al. 2003) is mounted on the ESO $3.6 \mathrm{~m}$ telescope, CORALIE (Queloz et al. 2001a) on the Swiss 1.2m Leonard Euler telescope, and FEROS (Kaufer \& Pasquini 1998) on the $2.2 \mathrm{~m}$ 


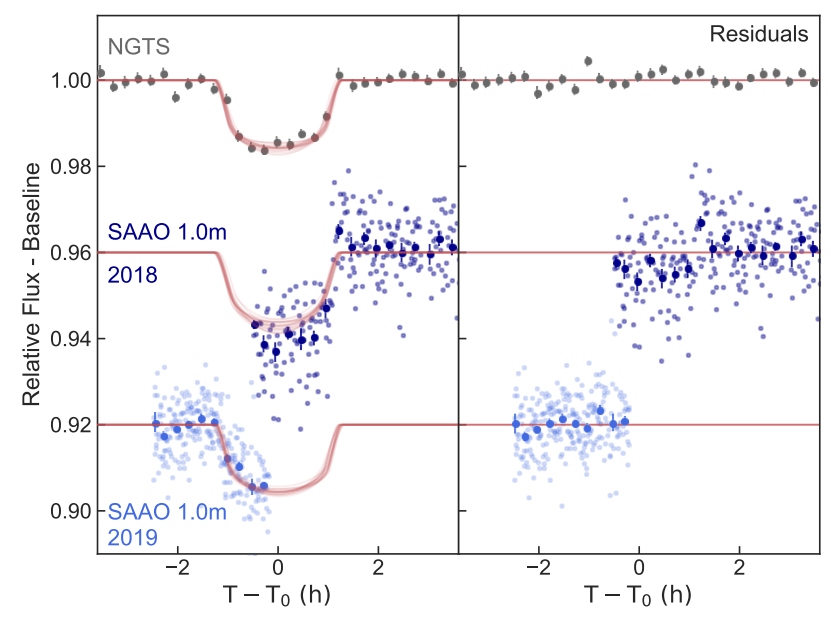

(a) Photometry for NGTS-15b. The NGTS discovery lightcurve is phasefolded at the best-fitting period of $3.27623 \pm 0.00001 \mathrm{~d}$. The lightcurves from the $1.0 \mathrm{~m}$ telescope at SAAO show a detection of egress from the $21 \mathrm{st}$ November 2018 and a detection of ingress from 6th of December 2019. Note that the in-transit scatter during the 2018 observation is a result of poor atmospheric conditions, and that the transit depths from global modelling for the NGTS and SAAO lightcurves agree to within errors.

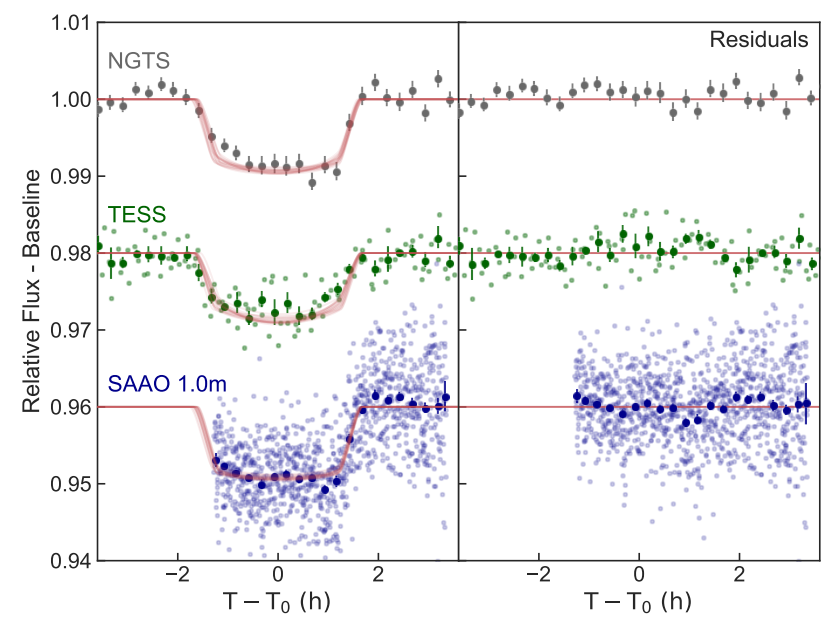

(c) Photometry for NGTS-17b. The NGTS discovery lightcurve and TESS follow-up lightcurve are phase-folded at the best-fitting period of $3.24253 \pm 0.00001 \mathrm{~d}$. The SAAO lightcurve consists of one dataset from 5 th February 2019.

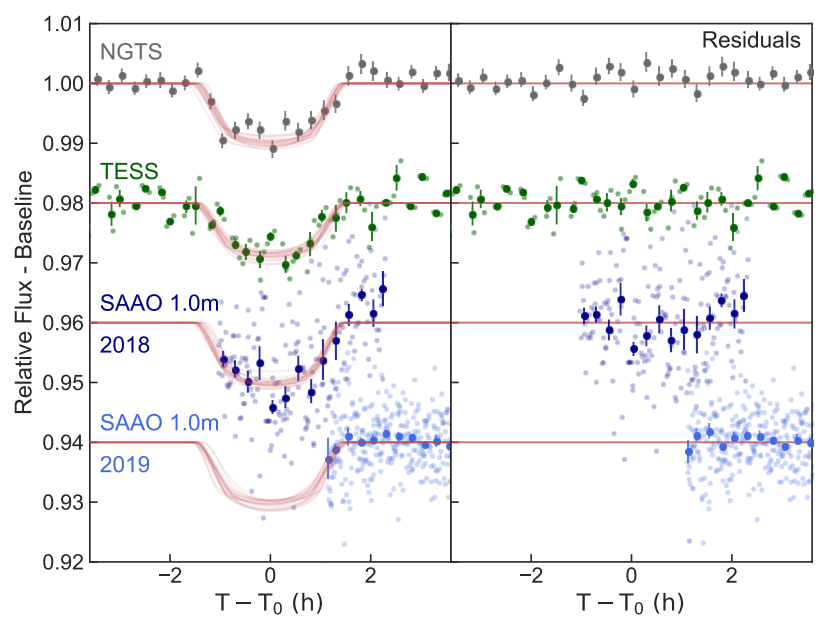

(b) Photometry for NGTS-16b. The NGTS and TESS lightcurves are phasefolded on the best fitting period, $4.84532 \pm 0.00002 \mathrm{~d}$. For this object, we obtained two lightcurves with the SAAO $1.0 \mathrm{~m}$ telescope on $21 \mathrm{st}$ December 2018 and 5th December 2019, both of which include egress. As with NGTS15 , the large in-transit scatter of the SAAO lightcurve from 2018 is due to poor atmospheric conditions and the true errors of these data points are likely underestimated. All four transit depths from the global modelling agree to within errors.

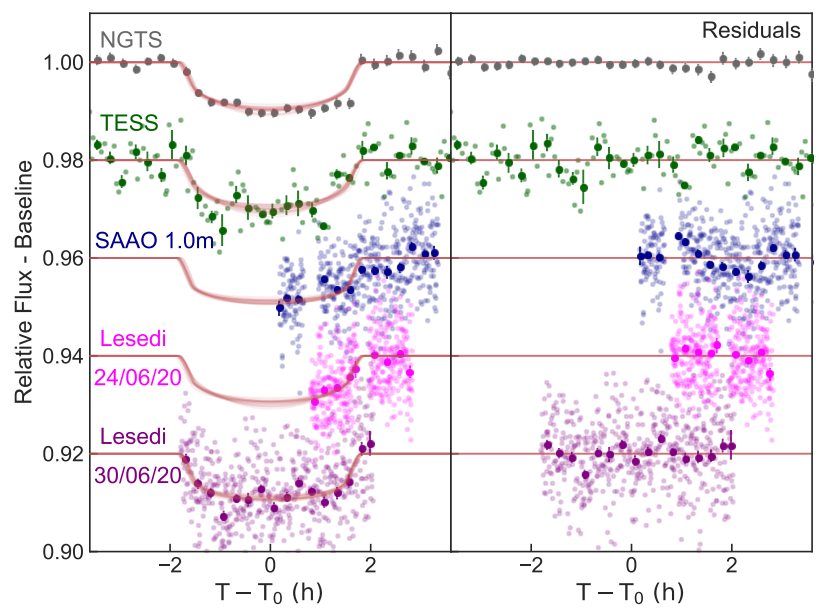

(d) Photometry for NGTS-18b. The NGTS discovery lightcurve and TESS follow-up lightcurve are phase-folded at the best-fitting period of $3.05125 \pm 0.00001 \mathrm{~d}$. The SAAO $1.0 \mathrm{~m}$ telescope lightcurve consists of one dataset from 21st March 2019; although egress is seen, due to the poor quality of the data, we omit it from our global modelling. The two Lesedi datasets in $I$ and $V$ band comprise of single observations from 24th and 30th June respectively.

Figure 1. NGTS discovery photometry (grey points) and follow up photometry (TESS: green points, SAAO $1.0 \mathrm{~m}$ telescope $I$ band: dark and light blue points, Lesedi $I$ band: pink points, Lesedi $V$ band: purple points) for each planet. Note that, for clarity, we exclude the unbinned NGTS data from the plots. The red lines show 20 lightcurve models generated from randomly drawn posterior samples of the ALLESFITTER fit. Residuals are shown to the right of the lightcurves.

MPG/ESO telescope. The spectrographs have a range of spectral resolutions of $\mathrm{R}=115,000, \mathrm{R}=60,000$ and $\mathrm{R}=48,000$, respectively. Due to the faintness of the targets $(14.3<\mathrm{V}<14.6$; see Tables $5,6,7$, and 8), all HARPS spectra were collected with the high efficiency fibre link (EGGS), which uses a fibre size of 1.4 " instead of the usual 1.0" mode. The HARPS-EGGS mode trades spectral resolution for approximately twice the photon count, depending on seeing. We used the second HARPS-EGGS fibre to monitor the sky simultaneously with science observations. All four objects were observed by FEROS and at least one other spectrograph, and we obtained a minimum of 12 data points for each (see Table 3 for details).

The HARPS data were reduced via the offline data reduction pipeline (DRS) before being cross-correlated with a binary G2 mask to extract radial velocities (Baranne et al. 1996). This procedure was also employed for the CORALIE data, for which the spectra were 
Table 3. Summary of radial velocity measurements. The full tables can be found in Appendix A.

\begin{tabular}{ccccccc}
\hline \hline \multirow{2}{*}{ Target } & Instrument & Nights observed & $\mathrm{N}_{\text {spectra }}$ & Exptime (s) & Programme & SNR $_{\text {combined }}$ \\
\hline \hline \multirow{2}{*}{ NGTS-15 } & CORALIE & $01 / 11 / 2018-01 / 03 / 2019$ & 7 & 2700 & N/A & - \\
& HARPS & $13 / 09 / 2019-03 / 12 / 2019$ & 7 & 1800 & $0103 . C-0719$ (A) \& 0104.C-0588(A) & 16.80 \\
& FEROS & $10 / 09 / 2019-19 / 09 / 2019$ & 7 & 1800 & $0103 . A-9004(\mathrm{~A})$ & - \\
\hline \multirow{2}{*}{ NGTS-16 } & HARPS & $21 / 01 / 2020-22 / 03 / 2020$ & 6 & 1800 & $0104 . C-0588(\mathrm{~A})$ & 21.77 \\
& FEROS & $30 / 12 / 2019-04 / 01 / 2020$ & 6 & 1800 & $0104 . A-9012(\mathrm{~A})$ & - \\
\hline \multirow{2}{*}{ NGTS-17 } & CORALIE & $01 / 10 / 2019-14 / 02 / 2020$ & 8 & 2700 & N/A & - \\
& FEROS & $09 / 11 / 2019-04 / 01 / 2020$ & 12 & 1800 & $0103 . A-9004(\mathrm{~A}) \& 0104 . A-9012(\mathrm{~A})$ & 59.00 \\
\hline \multirow{2}{*}{ NGTS-18 } & CORALIE & $21 / 05 / 2019-14 / 01 / 2020$ & 2 & 2700 & N/A & - \\
& HARPS & $02 / 03 / 2020-23 / 03 / 2020$ & 10 & $2400-2700$ & $0104 . C-0588(\mathrm{~A})$ & 26.69 \\
& FEROS & $30 / 12 / 2019-04 / 01 / 2020$ & 5 & 1800 & $0104 . A-9012(\mathrm{~A})$ & - \\
\hline
\end{tabular}

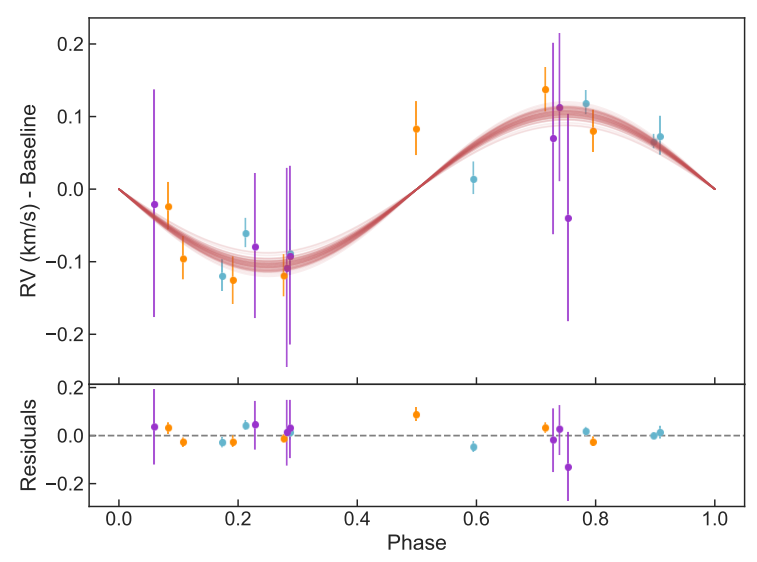

(a) NGTS-15

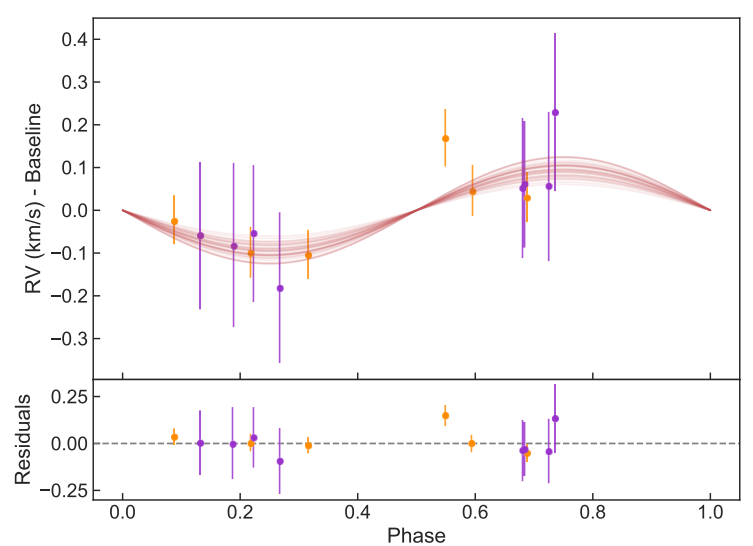

(c) NGTS-17

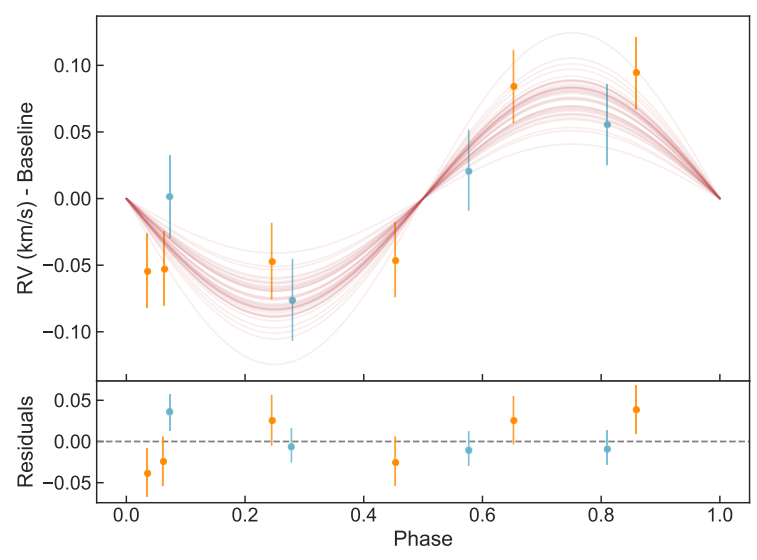

(b) NGTS-16

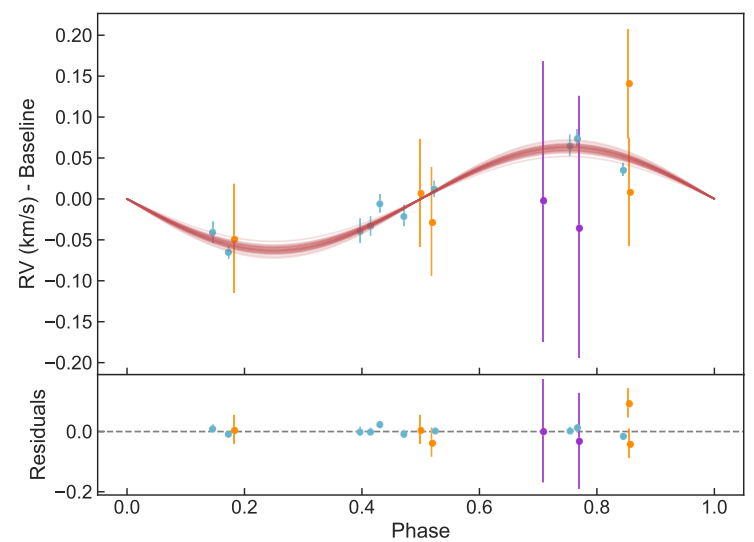

(d) NGTS-18

Figure 2. Phase-wrapped radial velocity measurements for all four stars from the HARPS (cyan points), FEROS (orange points) and CORALIE (purple points) spectrographs. The red lines show 20 radial velocity models generated from randomly drawn posterior samples of the ALLESFITTER fit.

reduced using the standard CORALIE DRS. For the data collected with FEROS, the CERES reduction pipeline (Brahm et al. 2017) performed a radial velocity extraction by the same method.

Two HARPS spectra of NGTS-18 obtained on BJD 2458916.780 and 2458918.843 were contaminated by moon light. We corrected the radial velocity measurements by subtracting the signal of the simultaneous sky-fibre from the science fibre in cross correlation function (CCF) space. The RVs are then extracted as per usual on the star-sky CCF. Additionally, the CORALIE radial velocities for NGTS-17 were computed while excluding the first 30 spectral orders, in which the signal-to-noise ratio was $<1$. Phasefolded plots of the radial velocity data for all four targets are shown in Figure 2.

For all four targets, the radial velocity measurements were con- 
sistent with a Jupiter-mass planet, with semi-amplitudes on the order of $\mathrm{K} \sim 100 \mathrm{~m} \mathrm{~s}^{-1}$ (see Table 9) and variations in-phase with the periods derived from the photometric data. To confirm that the signals did not arise due to cool stellar spots or a blended eclipsing binary, we checked for any correlation between the radial velocity measurements and the line bisector span of the CCF (Queloz et al. 2001b). Correspondingly, no evidence was found in the spectroscopic data that contradicted the existence of a planetary companion.

\section{ANALYSIS}

\subsection{Stellar Properties}

In order to produce reliable fits to our photometric and radial velocity data for NGTS-15, NGTS-16, NGTS-17 and NGTS-18, we must first determine the stellar properties of each system. Constraining these is vital as the accuracy of the measured planetary parameters is dependant on how well we can characterise the host star. Below we outline our stellar analysis procedure and results.

\subsubsection{Gaia DR2}

For all four objects, we obtained additional photometric and astrometric data from Gaia DR2 (Gaia Collaboration et al. 2016, 2018) (see Tables 5, 6, 7, and 8). Part of these data, including the stellar radius and parallax, were utilised as priors in our SED fitting procedure (see Section 3.1.3).

The Gaia parallaxes, proper motions, and absolute radial velocities of each star were used to determine the Galactic velocity components $\left(U_{L S R}, V_{L S R}, W_{L S R}\right)$, assuming a Local Standard of Rest of UVW $=(11.1,12.14,7.25) \mathrm{km} \mathrm{s}^{-1}$ (Schönrich et al. 2010). By using the selection criteria for kinematically thin-disk $\left(V_{\text {tot }}<50\right.$ $\left.\mathrm{kms}^{-2}\right)$ and kinematically thick-disk $\left(70<V_{\text {tot }}<180 \mathrm{kms}^{-2}\right)$ objects (Gaia Collaboration et al. 2018), we conclude that all four stars belong to the thin disk population (see Table 4).

Finally, our host stars show no evidence of being unresolved binaries, with all four targets having an astrometric excess noise of zero as well as a low $(<0)$ astrometric goodness of fit in the along-scan direction (GOF_AL) in Gaia DR2 (Evans 2018).

\subsubsection{Stellar analysis}

Although we used spectral energy distribution (SED) fitting to derive our final stellar parameters (see Section 3.1.3), we first fit the stacked spectra for each object in order to obtain suitable priors for the key parameters with which to constrain the SED fits. For this we used ISPEC (Blanco-Cuaresma et al. 2014; Blanco-Cuaresma 2019), an open-source framework for spectral analysis. ISPEC users can choose from a list of models for spectral synthesis; we employ the SPECTRUM (Gray \& Corbally 1994) radiative transfer code and the solar abundance model from Asplund et al. (2009). Additionally, we use the Gaia-ESO Survey (GES) line list (version 5.0 - Heiter et al. 2015), which covers the full wavelength range of our spectra (from 420-920nm), and the MARCES.GES model atmosphere (Gustafsson et al. 2008).

We used our high-resolution HARPS data for the spectral analysis of NGTS-15, NGTS-16, and NGTS-18. Since NGTS-17 was not observed with HARPS, we instead used the FEROS spectra. For each object, we shift the spectra to the laboratory frame of reference and co-add them to produce a single, high SNR, combined spectrum. These SNRs are reported in Table 3. We note that, although
NGTS-17 has the highest SNR, the data was affected by correlated red noise, which hampered spectral analysis.

We determined the stellar effective temperature, $T_{\mathrm{eff}}$, and the surface gravity, $\log g$, via fits to the the $\mathrm{H} \alpha, \mathrm{NaI} \mathrm{D}$ and $\mathrm{MgI} b$ lines. Individual FeI and FeII lines were used as diagnostics for metallicity $([\mathrm{Fe} / \mathrm{H}])$ and the rotational line-of-sight broadening $(v \sin i)$. Values for each parameter were obtained by synthesising model spectra until we found an acceptable fit to the data, and uncertainties were estimated by varying the fit until the models no longer matched the stacked spectra of each object.

From our spectral analysis, we find all four stars to be metalrich, which aligns with observations that short and intermediate period gas giants tend to be found around more metal-rich stars than longer period gas giants (Jenkins et al. 2017; Maldonado et al. 2019).

\subsubsection{SED fitting}

To determine the stellar parameters of each system, we performed a fit to their SEDs. For this, we employed the python tool ARIADNE (Vines \& Jenkins 2021), which fits catalogue photometry to different atmospheric model grids (Phoenix V2 (Husser et al. 2013), BTSettl, BT-Cond, BT-NextGen (Allard et al. 2012; Hauschildt et al. 1999), Castelli \& Kurucz (2004), Kurucz (1993)) which are then convolved with a range of filter response functions.

The SEDs were modelled by interpolating the model grids in $T_{\text {eff }}-\log g-[\mathrm{Fe} / \mathrm{H}]$ space, with radius, distance, and extinction in the $V$ band used as additional model parameters. Any underestimated uncertainties in the photometry were accounted for by including an individual excess noise term for each photometric dataset.

Priors for $T_{\text {eff }}, \log g$, and $[\mathrm{Fe} / \mathrm{H}]$ were taken from our stellar analysis with ISPEC, whilst priors for the stellar radius and distance were obtained from the Gaia DR2 values, although we used an inflated value for the radius error to account for modelling errors. In addition, $A_{V}$ was limited to the maximum line-of-sight value taken from the re-calibrated SFD galactic dust map (Schlegel et al. 1998; Schlafly \& Finkbeiner 2011). The priors for the excess noise parameters were set to a normal distribution with a mean of zero and a variance equal to five times the associated uncertainty.

The bayesian evidence for each model was calculated using the dynesty nested sampling package, which also produces the parameters' posterior samples (Speagle 2020). The relative probabilities of the models were then used as weights with which to calculate the weighted average of each parameter via the following equation:

$P\left(\theta_{i}\right)=\sum_{n=1}^{N} P\left(\theta \mid X, M_{n}\right) P\left(M_{n} \mid X\right)$

Where $\theta_{i}$ is the parameter to be averaged, $P\left(\theta \mid X, M_{n}\right)$ is the posterior distribution derived using Bayes Theorem, and $P\left(M_{n} \mid X\right)$ is the Bayesian evidence of the individual model.

Note that, by averaging over several posterior distributions, ARIADNE is able to achieve a higher precision than would typically be obtained with a single atmospheric model. Subsequently, some uncertainties may be underestimated. We therefore calculated an additional systematic error for these parameters by following the approach taken in Southworth et al. (2015), wherein each SED was fit with the individual stellar atmosphere models before being compared to the overall Bayesian model averaging solution. By taking the largest difference between the model values and the averaged 
Table 4. The galactic velocity components and total space velocities for NGTS-15 - 18.

\begin{tabular}{ccc}
\hline Target & $\left(U_{\boldsymbol{L S R}}, V_{\boldsymbol{L S R}}, W_{\boldsymbol{L} \boldsymbol{R}}\right)$ & $V_{\mathrm{tot}}\left(\mathrm{km} \mathrm{s}^{-1}\right)$ \\
\hline NGTS-15 & $(26.875 \pm 0.017,-10.106 \pm 0.024,-14.065 \pm 0.023)$ & 32 \\
NGTS-16 & $(23.193 \pm 0.004,-1.914 \pm 0.004,-15.158 \pm 0.007)$ & 28 \\
NGTS-17 & $(26.073 \pm 0.031,-10.465 \pm 0.046,-14.478 \pm 0.044)$ & 32 \\
NGTS-18 & $(9.385 \pm 0.008,7.917 \pm 0.020,9.548 \pm 0.011)$ & 16 \\
\hline
\end{tabular}

value from all posterior distributions, we were able to obtain the systematic uncertainty.

Finally, the mass value was estimated using MIST isochrones (Choi et al. 2016). The derived stellar parameters are listed in Tables 5, 6, 7 and 8. A detailed overview of the ARIADNE fitting procedure, as well as the accuracy and precision of the tool, can be found in Vines \& Jenkins (2021).

\subsection{Global Modelling}

We determined the physical parameters of the systems, including planet radius and mass, via a simultaneous fit to the photometric and spectroscopic data for each object. For this, we used the publicly available open-source astronomy software package ALLESFITTER (Günther \& Daylan 2020, 2019), which unites the packages ellc (light curve and RV models; Maxted 2016), emcee (MCMC sampling; Foreman-Mackey et al. 2013), and celerite (Gaussian Process (GP) models; Foreman-Mackey et al. 2017). The combination of these packages allows ALLESFITTER to model a variety of signals, including multi-star systems, star spots, stellar variability, and transit-timing variations.

Initial MCMC fits required millions of steps to converge, so we chose the Nested Sampling approach to produce our global fits. Our priors for transit epoch and transit depth $\left(R_{p} / R_{S}\right)$ were refined from ORION by generating quick MCMC fits to the NGTS data using BRUCE, an open-source binary star and exoplanet analysis package ${ }^{3}$. The results from the ARIADNE SED fits were used as priors on the stellar parameters, specifically $R_{S}, M_{S}$ and $T_{\text {eff }}$, although we used the $T_{\text {eff }}$ error from ISPEC as this is underestimated by ARIADNE. Because all four host stars show no out-of-transit variability or trends, GP modelling was not necessary; indeed, an initial GP fit to the 2019 SAAO data of NGTS-18 incorrectly adjusted a data gap between the mid-transit and egress data points, resulting in a smaller transit depth than the true value. Furthermore, we accounted for instrumental offsets in the radial velocity data, as each object was observed with at least two different spectrographs. We adopted a quadratic limb-darkening law as parameterised in Kipping (2013), and fit for the limb-darkening coefficients.

We ran two fits for each planet: one in which the orbital eccentricity, $e$, was fixed at 0 , and one for which $e$ was allowed to vary freely. Each of the latter fits resulted in non-zero values of $e$, at $0.102_{-0.068}^{+0.061}, 0.105_{-0.079}^{+0.130}, 0.168_{-0.101}^{+0.093}$, and $0.035_{-0.024}^{+0.033}$ for NGTS-15b, NGTS-16b, NGTS-17b, and NGTS-18b, respectively. However, Lucy \& Sweeney (1971) showed that many small values of $e$ are spurious, and define a probabilistic test to determine whether a small $e$ is statistically significant from 0 . By adopting a $5 \%$ level of significance, they find that if the condition $e>2.45 \sigma_{e}$ is satisfied (where $\sigma_{e}$ is the observational uncertainty on $e$ ), then one can be confident that the measured eccentricity is real. We find that all of

3 https://github.com/samgill844/bruce our measurements fail to meet this criteria, and we therefore adopt the results from the global modelling fits in which $e$ is fixed at zero.

We use ALLESFITTER to fit our data for the key physical parameters of each system, including the planet's orbital period $\mathrm{P}$, the radius ratio $R_{p} / R_{S}$, and the radial velocity semi-amplitude $\mathrm{K}$. Due to the large pixel scale of TESS (21" per pixel), the TESS FFI lightcurves for NGTS-16 and NGTS-17 contain additional faint (G z 17) neighbouring stars, and we therefore fit for a small dilution in the TESS data. We also note some blending for NGTS-15 in the NGTS data from a singular G 19 mag object (identified in Gaia $D R 2$ ), and account for this in our fitting. Note that dilution is defined in ALLESFITTER as:

$D=1-\frac{F_{\text {source }}}{F_{\text {source }}+F_{\text {blend }}}$

A full list of fitted properties and their values for each planet can be found in Table 9.

Figure 1 shows the phase-folded and single lightcurves for the NGTS, SAAO, and TESS data for each object, where the red lines indicate 20 models generated from randomly drawn posterior samples of the ALLESFITTER fits. Similarly, the phase-folded radial velocity data for each star is shown in Figure 2, with 20 generated radial velocity models.

\section{INFLATION}

All four planets presented in this paper have sub-Jovian masses and super-Jovian radii. Most notably, the radius of NGTS-18b is $1.21 \pm 0.18 R_{J}$, but with a mass of only $0.409_{-0.063}^{+0.081} M_{J}$. This is not unusual for close-in gaseous planets: the region of parameter space pertaining to low density hot Jupiters is well populated, and is driven by inflation mechanisms which correlate with stellar irradiation (Laughlin et al. 2011; Weiss et al. 2013; Thorngren \& Fortney 2018).

Studies by both Demory \& Seager (2011) and Miller \& Fortney (2011) suggest that these mechanisms become effective above an incident flux of $\sim 2 \times 10^{5} \mathrm{Wm}^{-2}$. By using the stellar luminosities and orbital parameters of each system, we calculated the irradiation received by each planet and found that all four are irradiated above this threshold (see Table 9 for values). As such, it is reasonable to expect that at least some of these planets may be affected by inflation processes and therefore exhibit radii which are larger than predicted.

To parameterise this, we follow the procedure outlined in Costes et al. (2020), which utilises the work of Baraffe et al. (2008) to compare planetary radii with predictions from evolutionary models, and the work of Sestovic et al. (2018) to discern the predicted additional radius change from inflation, $\Delta R$.

Baraffe et al. (2008) (hereafter B08) produced theoretical planetary evolution models which account for uncertainties in previous 
Table 5. Stellar Properties for NGTS-15. For the values from ARIADNE, we include a second systematic error from the difference between the average best-fit value and the maximum value from the individual stellar atmosphere models.

\begin{tabular}{lcc}
\hline Property & Value & Source \\
\hline 2MASS I.D. & $04532526-3248011$ & 2MASS \\
Gaia source I.D. & 4873830691665395584 & Gaia DR2 \\
TIC I.D. & TIC-1333933 & TIC8
\end{tabular}

Astrometric Properties

R.A.

Dec

$\mu_{\text {R.A. }}\left(\right.$ mas y $\left.^{-1}\right)$

$\mu_{\text {Dec. }}\left(\right.$ mas $\left.^{-1}\right)$

Parallax (mas)

Photometric Properties

V (mag)
B (mag)
g (mag)
r (mag)
i (mag)
G (mag)
J (mag)
H (mag)
K (mag)
W1 (mag)
W2 (mag)
T (mag)

Derived Properties

Spectral type

$\mathrm{T}_{\text {eff }}(\mathrm{K})$

$[\mathrm{Fe} / \mathrm{H}]$

vsini $\left(\mathrm{km} \mathrm{s}^{-1}\right)$

$\gamma_{R V}\left(\mathrm{~km} \mathrm{~s}^{-1}\right)$

$\log \mathrm{g}$

$M_{S}\left(M_{\odot}\right)$

$R_{S}\left(R_{\odot}\right)$

Age (Gyrs)

Distance (pc)

$A_{v}$ (mag)

$\begin{array}{ll}04^{\mathrm{h}} 53^{\mathrm{m}} 25^{\mathrm{s}} .27 & \text { Gaia DR2 } \\ -32^{\circ} 48^{\prime} 01^{\prime \prime} 25 & \text { Gaia DR2 } \\ 5.687 \pm 0.027 & \text { Gaia DR2 } \\ 1.316 \pm 0.035 & \text { Gaia DR2 } \\ 1.242 \pm 0.017 & \text { Gaia DR2 }\end{array}$

APASS

APASS

APASS

APASS

APASS

Gaia DR2

2MASS

2MASS

2MASS

WISE

WISE

TIC8
Table 6. Stellar Properties for NGTS-16. Systematic uncertainties are included on the values from ARIADNE as in Table 5.

\begin{tabular}{lcc}
\hline Property & Value & Source \\
\hline 2MASS I.D. & $03530331-3048164$ & 2MASS \\
Gaia source I.D. & 4886825544715697792 & Gaia DR2 \\
TIC I.D. & TIC-166806344 & TIC8
\end{tabular}

Astrometric Properties

$\begin{array}{lcc}\text { R.A. } & 03^{\mathrm{h}} 53^{\mathrm{m}} 03^{\mathrm{s}} .34 & \text { Gaia DR2 } \\ \text { Dec } & -30^{\circ} 48^{\prime} 16^{\prime \prime} 71 & \text { Gaia DR2 } \\ \mu_{\text {R.A. }\left(\mathrm{mas} \mathrm{y}^{-1}\right)} & 9.772 \pm 0.031 & \text { Gaia DR2 } \\ \mu_{\text {Dec. }}\left(\mathrm{mas} \mathrm{y}^{-1}\right) & -4.673 \pm 0.043 & \text { Gaia DR2 } \\ \text { Parallax (mas) } & 1.084 \pm 0.021 & \text { Gaia DR2 }\end{array}$

Photometric Properties

$\begin{array}{lcc}\text { V (mag) } & 14.364 \pm 0.039 & \text { APASS } \\ \text { B (mag) } & 15.140 \pm 0.006 & \text { APASS } \\ \text { g (mag) } & 14.743 \pm 0.012 & \text { APASS } \\ \text { r (mag) } & 14.148 \pm 0.018 & \text { APASS } \\ \text { i (mag) } & 13.960 \pm 0.078 & \text { APASS } \\ \text { G (mag) } & 14.2311 \pm 0.0002 & \text { Gaia DR2 } \\ \text { J (mag) } & 13.090 \pm 0.024 & \text { 2MASS } \\ \text { H (mag) } & 12.735 \pm 0.027 & \text { 2MASS } \\ \text { K (mag) } & 12.647 \pm 0.033 & \text { 2MASS } \\ \text { W1 (mag) } & 12.638 \pm 0.025 & \text { WISE } \\ \text { W2 (mag) } & 12.710 \pm 0.025 & \text { WISE } \\ \text { T (mag) } & 13.743 \pm 0.007 & \text { TIC8 }\end{array}$

Derived Properties

Spectral type

$\mathrm{T}_{\text {eff }}(\mathrm{K})$

[Fe/H]

vsini $\left(\mathrm{km} \mathrm{s}^{-1}\right)$

$\gamma_{R V}\left(\mathrm{~km} \mathrm{~s}^{-1}\right)$

$\log \mathrm{g}$

$M_{S}\left(M_{\odot}\right)$

$R_{S}\left(R_{\odot}\right)$

Age (Gyrs)

Distance (pc)

$A_{v}$ (mag)

$\begin{array}{cc}\text { G7V } & \text { ARIADNE } \\ 5550 \pm 150 & \text { ISPEC } \\ 0.35 \pm 0.1 & \text { ISPEC } \\ 2.5 \pm 0.8 & \text { ISPEC } \\ 29.13 \pm 0.01 & \text { RV data } \\ 4.35_{-0.08}^{+0.11} \pm 0.14 & \text { ARIADNE } \\ 1.002 \pm 0.011 \pm 0.029 & \text { ARIADNE } \\ 1.213_{-0.032}^{+0.040} \pm 0.102 & \text { ARIADNE } \\ 10.29_{-0.53}^{+0.74} \pm 1.38 & \text { SED fitting } \\ 892_{-23}^{+29} \pm 63 & \text { ARIADNE } \\ 0.013 \pm 0.004 \pm 0.011 & \text { ARIADNE }\end{array}$

2MASS (Skrutskie et al. 2006); APASS (Henden \& Munari 2014); WISE (Wright et al. 2010); Gaia (Gaia Collaboration et al. 2016);

TIC8 (Stassun et al. 2019)

TIC8 (Stassun et al. 2019) models, most notably quantifying the impact of heavy element enrichment. Their results include tables of predicted planetary radii which vary with the system's physical parameters. Using these tables and adjusting for spectral type, we calculated the expected radii for all four planets and compared the result with the radii derived from our global fits. A discrepancy between the two values could indicate that inflation mechanisms have affected the radius. As B08 account for a decrease in radius with increasing heavy element mass, we assume a mass fraction of heavy material of $0.02-0.1$ for each planet, which provides a realistic upper limit for the non-inflated radii.

Sestovic et al. (2018) (hereafter S18) implement hierarchical Bayesian modelling and a forward model to infer relationships between incident flux, radius, and mass using a population of 286 hot Jupiters with measured radii and masses. The resulting relations for $\Delta R$ vary according to four different mass regimes: below 
Table 7. Stellar Properties for NGTS-17. Systematic uncertainties are included on the values from ARIADNE as in Table 5.

\begin{tabular}{lcc}
\hline Property & Value & Source \\
\hline 2MASS I.D. & $04513613-3413342$ & 2MASS \\
Gaia source I.D. & 4873225513593736960 & Gaia DR2 \\
TIC I.D. & TIC-1309019 & TIC8
\end{tabular}

Astrometric Properties

$\begin{array}{lcc}\text { R.A. } & 04^{\mathrm{h}} 51^{\mathrm{m}} 36.14 & \text { Gaia DR2 } \\ \text { Dec } & -34^{\circ} 13^{\prime} 34^{\prime \prime} 37 & \text { Gaia DR2 } \\ \mu_{\text {R.A. }}\left(\mathrm{mas} \mathrm{y}^{-1}\right) & -0.190 \pm 0.031 & \text { Gaia DR2 } \\ \mu_{\text {Dec. }}\left(\mathrm{mas} \mathrm{y}^{-1}\right) & -11.812 \pm 0.034 & \text { Gaia DR2 } \\ \text { Parallax (mas) } & 0.932 \pm 0.020 & \text { Gaia DR2 }\end{array}$

Photometric Properties

$\begin{array}{lcc}\text { V (mag) } & 14.326 \pm 0.030 & \text { APASS } \\ \text { B (mag) } & 15.043 \pm 0.033 & \text { APASS } \\ \text { g (mag) } & 14.626 \pm 0.013 & \text { APASS } \\ \text { r (mag) } & 14.161 \pm 0.043 & \text { APASS } \\ \text { i (mag) } & 13.998 \pm 0.081 & \text { APASS } \\ \text { G (mag) } & 14.2136 \pm 0.0004 & \text { Gaia DR } \\ \text { J (mag) } & 13.114 \pm 0.026 & \text { 2MASS } \\ \text { H (mag) } & 12.867 \pm 0.027 & \text { 2MASS } \\ \text { K (mag) } & 12.724 \pm 0.029 & \text { 2MASS } \\ \text { W1 (mag) } & 12.739 \pm 0.023 & \text { WISE } \\ \text { W2 (mag) } & 12.747 \pm 0.027 & \text { WISE } \\ \text { T (mag) } & 13.767 \pm 0.006 & \text { TIC8 }\end{array}$

Derived Properties

$\begin{array}{lcc}\text { Spectral type } & \mathrm{G} 4 \mathrm{~V} & \text { ARIADNE } \\ \mathrm{T}_{\text {eff }}(\mathrm{K}) & 5650 \pm 100 & \text { ISPEC } \\ {[\mathrm{Fe} / \mathrm{H}]} & 0.15 \pm 0.1 & \text { ISPEC } \\ \operatorname{vsini}\left(\mathrm{km} \mathrm{s}^{-1}\right) & 4.1 \pm 1.0 & \text { ISPEC } \\ \gamma_{R V}\left(\mathrm{~km} \mathrm{~s}^{-1}\right) & 34.81 \pm 0.07 & \text { RV data } \\ \log \mathrm{g} & 4.00 \pm 0.09 \pm 0.08 & \text { ARIADNE } \\ M_{S}\left(M_{\odot}\right) & 1.025_{-0.014}^{+0.015} \pm 0.03 & \text { ARIADNE } \\ R_{S}\left(R_{\odot}\right) & 1.337_{0.038} \pm 0.119 & \text { ARIADNE } \\ \text { Age }(\mathrm{Gyrs}) & 9.22_{-0.48}^{+0.49} \pm 1.32 & \text { SED fitting } \\ \text { Distance }(\mathrm{pc}) & 1047_{-27}^{29} \pm 108 & \text { ARIADNE } \\ A_{v}(\mathrm{mag}) & 0.028 \pm 0.008 \pm 0.019 & \text { ARIADNE }\end{array}$

2MASS (Skrutskie et al. 2006); APASS (Henden \& Munari 2014); WISE (Wright et al. 2010); Gaia (Gaia Collaboration et al. 2016);

TIC8 (Stassun et al. 2019)

$0.37 M_{J}$, between $0.37-0.98 M_{J}$, between $0.98-2.50 M_{J}$ and over $2.50 M_{J}$. In this case, all four of the planets fall into the same mass range $\left(0.37-0.98 M_{J}\right)$, and we therefore use the corresponding equation to derive the expected radius increase due to inflation for each:

$\Delta R=0.70 \cdot\left(\log _{10} F-5.5\right), \quad 0.37 \leq \frac{M}{M_{J}}<0.98$

(see S18 Equation 11). Note that, in the S18 models, the 'baseline' radius, $R$, is set to $0.98 \pm 0.04 R_{J}$ for this mass regime. We therefore
Table 8. Stellar Properties for NGTS-18. Systematic uncertainties are included on the values from ARIADNE as in Table 5.

\begin{tabular}{lcc}
\hline Property & Value & Source \\
\hline 2MASS I.D. & $12021109-3532550$ & 2MASS \\
Gaia source I.D. & 3462511310147530752 & Gaia DR2 \\
TIC I.D. & TIC-142211778 & TIC8
\end{tabular}

Astrometric Properties

$\begin{array}{lcc}\text { R.A. } & 12^{\mathrm{h}} 02^{\mathrm{m}} 111^{\mathrm{s}} .09 & \text { Gaia DR2 } \\ \text { Dec } & -35^{\circ} 32^{\prime} 54^{\prime \prime} 99 & \text { Gaia DR2 } \\ \mu_{\text {R.A. }\left(\text { mas y }^{-1}\right)} & -2.416 \pm 0.040 & \text { Gaia DR2 } \\ \mu_{\text {Dec. }}\left(\text { mas y }^{-1}\right) & 1.487 \pm 0.025 & \text { Gaia DR2 } \\ \text { Parallax (mas) } & 0.884 \pm 0.034 & \text { Gaia DR2 }\end{array}$

Photometric Properties

$\begin{array}{lcc}\text { V (mag) } & 14.540 \pm 0.038 & \text { APASS } \\ \text { B (mag) } & 15.322 \pm 0.046 & \text { APASS } \\ \text { g (mag) } & 14.871 \pm 0.021 & \text { APASS } \\ \text { r (mag) } & 14.387 \pm 0.080 & \text { APASS } \\ \text { i (mag) } & 14.189 \pm 0.122 & \text { APASS } \\ \text { G (mag) } & 14.3896 \pm 0.0003 & \text { Gaia DR2 } \\ \text { J (mag) } & 13.265 \pm 0.030 & \text { 2MASS } \\ \text { H (mag) } & 12.888 \pm 0.023 & \text { 2MASS } \\ \text { K (mag) } & 12.870 \pm 0.032 & \text { 2MASS } \\ \text { W1 (mag) } & 12.768 \pm 0.023 & \text { WISE } \\ \text { W2 (mag) } & 12.832 \pm 0.026 & \text { WISE } \\ \text { T (mag) } & 13.907 \pm 0.006 & \text { TIC8 }\end{array}$

Derived Properties

Spectral type

$\mathrm{T}_{\text {eff }}(\mathrm{K})$

$[\mathrm{Fe} / \mathrm{H}]$

vsini $\left(\mathrm{km} \mathrm{s}^{-1}\right)$

$\gamma_{R V}\left(\mathrm{~km} \mathrm{~s}^{-1}\right)$

$\log \mathrm{g}$

$M_{S}\left(M_{\odot}\right)$

$R_{S}\left(R_{\odot}\right)$

Age (Gyrs)

Distance (pc)

$\begin{array}{cc}\text { G5V } & \text { ARIADNE } \\ 5610 \pm 150 & \text { ISPEC } \\ 0.15 \pm 0.1 & \text { ISPEC } \\ <0.5 & \text { ISPEC } \\ 5.19 \pm 0.02 & \text { RV data } \\ 4.16 \pm 0.05 \pm 0.05 & \text { ARIADNE } \\ 1.003_{-0.012}^{+0.020} \pm 0.022 & \text { ARIADNE } \\ 1.392_{-0.058}^{+0.057} \pm 0.201 & \text { ARIADNE } \\ 10.84_{-0.78}^{+0.40} \pm 1.22 & \text { SED fitting } \\ 1108_{-47}^{+44} \pm 158 & \text { ARIADNE } \\ 0.11 \pm 0.028 \pm 0.022 & \text { ARIADNE }\end{array}$

2MASS (Skrutskie et al. 2006); APASS (Henden \& Munari 2014); WISE (Wright et al. 2010); Gaia (Gaia Collaboration et al. 2016);

TIC8 (Stassun et al. 2019)

calculate the total expected inflated radius from the models of S18 as

$R_{\text {Inflated }}=0.98+\Delta R$

and compare this to the 'true' radii of the planets derived from global modelling. 
Table 9. Planetary properties for each system from ALLESFITTER (see Equation 2 for a definition of dilution, D)

(a) Planetary Properties for NGTS-15b

\begin{tabular}{lc}
\hline Property & Value \\
\hline $\mathrm{P}$ (days) & $3.27623 \pm 0.00001$ \\
$\mathrm{~T}_{C}$ (BJD) & $2458405.0558 \pm 0.0007$ \\
$\mathrm{~T}_{14}$ (hours) & $2.504_{-0.058}^{+0.060}$ \\
$R_{p} / R_{*}$ & $0.119^{ \pm 0.003}$ \\
$a / R_{*}$ & $10.00 \pm 0.59$ \\
$b$ & $0.509_{-0.112}^{+0.086}$ \\
$\mathrm{~K}\left(\mathrm{~m} \mathrm{~s}^{-1}\right)$ & $106^{-7}$ \\
$\mathrm{e}$ & $0($ fixed $)$ \\
$M_{p}\left(M_{J}\right)$ & $0.751_{-0.088}^{+0.102}$ \\
$R_{p}\left(R_{J}\right)$ & $1.10 \pm 0.10$ \\
$\rho_{p}\left(\mathrm{~g} \mathrm{~cm}^{-3}\right)$ & $0.74_{-0.10}^{+0.11}$ \\
$\mathrm{a}(\mathrm{AU})$ & $0.0441_{0.0046}$ \\
$\mathrm{~T}_{e q}(\mathrm{~K})$ & $1146 \pm 47$ \\
Irradiation $\left(\mathrm{Wm}^{-2}\right)$ & $(5.78 \pm 1.33) \times 10^{5}$ \\
$\mathrm{D}_{\mathrm{NGTS}}$ & $0.04_{-0.02}^{+0.01}$ \\
\hline
\end{tabular}

(c) Planetary Properties for NGTS-17b

\begin{tabular}{lc}
\hline Property & Value \\
\hline $\mathrm{P}$ (days) & $3.24253 \pm 0.00001$ \\
$\mathrm{~T}_{C}(\mathrm{BJD})$ & $2458442.5219 \pm 0.0009$ \\
$\mathrm{~T}_{14}$ (hours) & $3.391_{-0.073}^{+0.076}$ \\
$R_{p} / R_{*}$ & $0.095^{0.001}$ \\
$a / R_{*}$ & $6.28 \pm 0.40$ \\
$b$ & $0.688_{-0.056}^{+0.043}$ \\
$\mathrm{~K}\left(\mathrm{~m} \mathrm{~s}^{-1}\right)$ & $93_{-17}^{+20}$ \\
$\mathrm{e}$ & $0($ fixed $)$ \\
$M_{p}\left(M_{J}\right)$ & $0.764_{-0.164}^{+0.195}$ \\
$R_{p}\left(R_{J}\right)$ & $1.24 \pm 0.11$ \\
$\rho_{p}\left(\mathrm{~g} \mathrm{~cm}^{-3}\right)$ & $0.50_{-0.17}^{+0.27}$ \\
$\mathrm{a}(\mathrm{AU})$ & $0.0391 \pm 0.0043$ \\
$\mathrm{~T}_{e q}(\mathrm{~K})$ & $1457 \pm 50$ \\
Irradiation $\left(\mathrm{Wm}^{-2}\right)$ & $(1.58 \pm 0.34) \times 10^{6}$ \\
$\mathrm{D}_{\mathrm{TESS}}$ & $0.09_{-0.02}^{+0.01}$ \\
\hline
\end{tabular}

(b) Planetary Properties for NGTS-16b

\begin{tabular}{lc}
\hline Property & Value \\
\hline $\mathrm{P}$ (days) & $4.84532 \pm 0.00002$ \\
$\mathrm{~T}_{C}$ (BJD) & $2458435.6054 \pm 0.0013$ \\
$\mathrm{~T}_{14}$ (hours) & $3.061_{-0.136}^{+0.143}$ \\
$R_{p} / R_{*}$ & $0.110 \pm 0.005$ \\
$a / R_{*}$ & $9.29 \pm 0.83$ \\
$b$ & $0.807_{0.036}^{+0.049}$ \\
$\mathrm{~K}\left(\mathrm{~m} \mathrm{~s}^{-1}\right)$ & $74_{-12}^{+14}$ \\
$\mathrm{e}$ & $0(\mathrm{fixed})$ \\
$M_{p}\left(M_{J}\right)$ & $0.667_{-0.129}^{+0.157}$ \\
$R_{p}\left(R_{J}\right)$ & $1.30_{-0.12}^{+0.13}$ \\
$\rho_{p}\left(\mathrm{~g} \mathrm{~cm}^{-3}\right)$ & $0.38_{-0.12}^{+0.19}$ \\
$\mathrm{a}(\mathrm{AU})$ & $0.0523 \pm 0.0064$ \\
$\mathrm{~T}_{e q}(\mathrm{~K})$ & $1177 \pm 59$ \\
$\mathrm{Irradiation}\left(\mathrm{Wm}^{-2}\right)$ & $(6.60 \pm 1.56) \times 10^{5}$ \\
$\mathrm{D}_{\mathrm{TESS}}$ & $0.11_{-0.05}^{+0.03}$ \\
\hline
\end{tabular}

(d) Planetary Properties for NGTS-18b

\begin{tabular}{lc}
\hline Property & Value \\
\hline $\mathrm{P}$ (days) & $3.05125 \pm 0.00001$ \\
$\mathrm{~T}_{C}$ (BJD) & $2458564.4506 \pm 0.0007$ \\
$\mathrm{~T}_{14}$ (hours) & $3.601_{-0.047}^{+0.055}$ \\
$R_{p} / R_{*}$ & $0.089 \pm 0.001$ \\
$a / R_{*}$ & $6.97 \pm 0.27$ \\
$b$ & $0.183_{-0.115}^{+0.147}$ \\
$\mathrm{~K}\left(\mathrm{~m} \mathrm{~s}^{-1}\right)$ & $62 \pm 5$ \\
$\mathrm{e}$ & $0($ fixed $)$ \\
$M_{p}\left(M_{J}\right)$ & $0.409_{-0.063}^{+0.081}$ \\
$R_{p}\left(R_{J}\right)$ & $1.21 \pm 0.18$ \\
$\rho_{p}\left(\mathrm{~g} \mathrm{~cm}^{-3}\right)$ & $0.28_{-0.12}^{+0.25}$ \\
$\mathrm{a}(\mathrm{AU})$ & $0.0448 \pm 0.0068$ \\
$\mathrm{~T}_{e q}(\mathrm{~K})^{\dagger}$ & $1381_{-53}^{+55}$ \\
Irradiation $\left(\mathrm{Wm}^{-2}\right)$ & $(1.15 \pm 0.37) \times 10^{6}$ \\
\hline
\end{tabular}

${ }^{\dagger} \mathrm{T}_{e q}=\mathrm{T}_{\mathrm{eff} ; s} \cdot \frac{(1-A)^{1 / 4}}{E} \cdot \sqrt{\frac{R_{s}}{2 a}}$, where albedo $A=0.3$ and emissivity $E=1$

\section{DISCUSSION}

We present our inflation results in Table 10, and summarise below:

NGTS-15b: Due to the poorly constrained age for this planet, the range of possible values for $R_{\text {non-inflated }}$ is so broad that it encompasses and exceeds the expected $R_{\text {inflated values. As }}$ such, $R_{\text {observed }}$ and its uncertainties appear consistent with both inflated and non-inflated radii, and we are therefore unable to draw firm conclusions about the nature of inflation of NGTS- $15 \mathrm{~b}$.

NGTS-16b: $R_{\text {observed }}$ does not agree to within uncertainties with $R_{\text {non-inflated }}$, but it is consistent with $R_{\text {inflated }}$. However, the discrepancy between $R_{\text {observed }}$ and $R_{\text {non-inflated }}$ is less than $2 \sigma$, so the possibility that this planet is not inflated is also non-negligable.

NGTS-17b: We find that, whilst $R_{\text {observed }}$ is higher than $R_{\text {non-inflated }}$, it is not as large as $R_{\text {inflated }}$. Although the discrepancy 
Table 10. Quantifying the inflation of NGTS-15b, NGTS-16b, NGTS-17b, and NGTS-18b. $R_{\text {observed }}$ describes the radius derived from global fits to the observational data, whilst $R_{\text {non-inflated }}$ and $R_{\text {inflated }}$ describe the predicted radius from non-inflationary evolutionary models and inflationary forward models, respectively.

\begin{tabular}{ccccccc}
\hline \hline Target & Irradiation & Mass $\left(M_{J}\right)$ & $\Delta R\left(R_{J}\right)^{*}$ & $R_{\text {inflated }}\left(R_{J}\right)^{*}$ & $R_{\text {non-inflated }}\left(R_{J}\right)^{* *}$ & $R_{\text {observed }}\left(R_{J}\right)$ \\
\hline \hline NGTS-15b & $(5.78 \pm 1.33) \times 10^{5}$ & $0.751_{-0.088}^{+0.102}$ & $0.18 \pm 0.07$ & $1.16 \pm 0.08$ & $1.02-1.45$ & $1.10 \pm 0.10$ \\
\hline NGTS-16b & $(6.60 \pm 1.56) \times 10^{5}$ & $0.667_{-0.129}^{+0.157}$ & $0.22 \pm 0.07$ & $1.20 \pm 0.08$ & $1.01-1.07$ & $1.30_{-0.12}^{+0.13}$ \\
\hline NGTS-17b & $(1.58 \pm 0.34) \times 10^{6}$ & $0.764_{-0.164}^{+0.195}$ & $0.49 \pm 0.07$ & $1.47 \pm 0.08$ & $1.01-1.08$ & $1.24 \pm 0.11$ \\
\hline NGTS-18b & $(1.15 \pm 0.37) \times 10^{6}$ & $0.409_{-0.063}^{+0.081}$ & $0.40 \pm 0.10$ & $1.38 \pm 0.11$ & $0.99-1.06$ & $1.21 \pm 0.18$ \\
\hline
\end{tabular}

*From Sestovic et al. (2018); **From Baraffe et al. (2008)

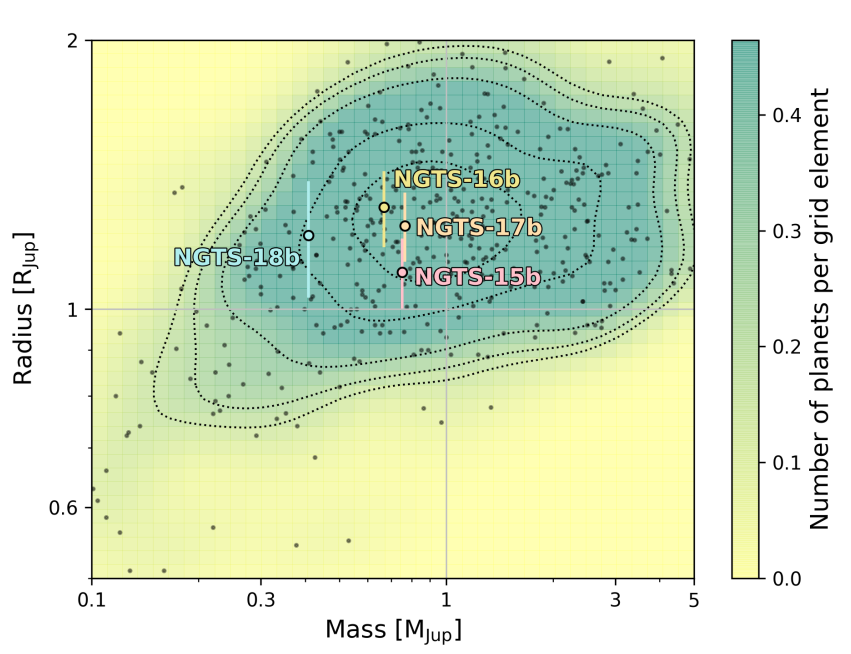

Figure 3. Exoplanets with confirmed masses and radii (grey points) from the NASA Exoplanet Archive (https://exoplanetarchive.ipac. caltech.edu/). The background and the dotted black contour lines highlight the point density per grid element of our sample. The hot Jupiters presented in this paper are plotted with error bars, and can be seen to lie comfortably within the general population of hot Jupiters. NGTS-15b to NGTS-18b are labeled with coloured circles on the plot with associated uncertainties.

between $R_{\text {observed }}$ and $R_{\text {non-inflated }}$ is less than $2 \sigma$, note that, due to the high irradiation of NGTS-17b, the planet exists in a region of parameter space in which S18 find that there is no evidence for a population of non-inflated hot Jupiters. It is therefore probable that this planet is inflated and that the models from S18 fail to predict the true radius value.

NGTS-18b: For this planet, the large uncertainties of $R_{\text {observed }}$ mean that NGTS-18b can be described by both $R_{\text {inflated }}$ and $R_{\text {non-inflated }}$; although as with NGTS-17b, we note that the large incident flux implies that this planet is likely to be inflated. In addition, we find that the lower boundary of $R_{\text {observed }}$ falls into S18's low-mass regime (below $0.37 M_{J}$ ), and we therefore use the corresponding equation for $\Delta R$ from S18 to derive an alternative $R_{\text {inflated }}$ value of $0.96 \pm 0.9$. In this case, again $R_{\text {observed }}$ is in agreement with $R_{\text {inflated }}$ to within errors, but now $R_{\text {inflated }}$ and $R_{\text {non-inflated }}$ are also entirely consistent with one another, leading us inclined to disregard this result.

Although all of the incident fluxes are larger than the typically accepted threshold for inflation $\left(2 \times 10^{5} \mathrm{Wm}^{-2}\right)$ (Demory \& Seager

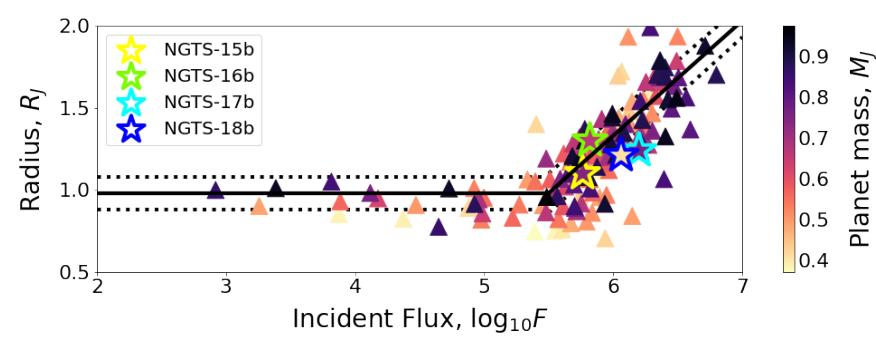

Figure 4. Exoplanets from the Exoplanet Orbit Database (Han et al. 2014) with masses between $0.37-0.97 M_{\boldsymbol{J}}$. We overplot the model (solid black line) from Sestovic et al. (2018) with the associated standard deviation in $R$ (dotted black line) for hot Jupiter inflation in this mass regime. The hot Jupiters presented in this paper are plotted as stars with coloured outlines.

2011; Miller \& Fortney 2011), it is possible that some of these planets, particularly NGTS-15b, are not inflated. However, this boundary only describes the minimum limit at which planets begin to appear inflated, and so this result is not unexpected. In addition, S18 finds that above $\sim 10^{6} \mathrm{Wm}^{-2}$, there is no evidence for a population of non-inflated hot Jupiters in the mass range $0.37-0.98 M_{J}$. The results presented here do not necessarily support this claim, but as the two planets experiencing irradiation in excess of $\sim 10^{6} \mathrm{Wm}^{-2}$ do show some evidence for inflation, we take the results from S18 as an indication that NGTS-17b and NGTS-18b are more likely to be inflated than not.

It is interesting that, based on the models of S18, we expect both NGTS-15b and NGTS-16b to possess a similarly inflated radius. Whilst it is possible that both planets do correspond with the predicted inflated radii from S18, it is also probable that NGTS-15b is not inflated at all, especially if the system is young. However, we note that, whilst S18's model successfully describes the general shape of the hot Jupiter population in the radius-flux plane (see Figure 4), the variation in latent parameters such as heavy element fraction and system age is only accounted for in the scatter of the model; currently, the models are solely dependant on incident flux (within mass bins). We speculate that the variation in additional latent factors for the planets presented here would provide an explanation as to why two similarly irradiated planets may exhibit different levels of radius inflation. As previously mentioned, the age of NGTS-15b could reasonably lead to an uninflated radius; it is already known that hot Jupiter radii vary with age (Baraffe et al. 2008; Miller et al. 2009; Thorngren \& Fortney 2018), and new evidence is emerging which suggests that gaseous planets may 'reinflate' at late times (Hartman et al. 2016; Komacek et al. 2020; Thorngren et al. 2020, 2021). In addition, differing system ages could indicate 
a difference in planetary migration times onto short orbits, and there may have been a variation in the level of stellar irradiation since that time. This may be one explanation for the systems presented here, as the SED ages, while uncertain for the younger system, would appear to align with this. Furthermore, as noted previously, the fraction of heavy elements present in a planet will influence the radius inflation of hot Jupiters, with radii expected to decrease as $Z$ increases (Thorngren et al. 2016, 2021).

Whilst these parameters are described indirectly by the models of S18 as the intrinsic physical scatter in the data, the detailed effects are as-of-yet unidentified. With the number of hot Jupiter candidates increasing more than two fold since the construction of the sample from S18, it would be pertinent to update these models with a view to understanding whether the inclusion of additional hyperparameters in the Bayesian model may describe the hot Jupiter inflation relationships with greater accuracy.

Finally, despite the varying response to inflation mechanisms, we find that all four planets lie comfortably amongst the general population of hot Jupiters in the mass-radius plane (see Figure 3 ). Additionally, all four planets again occupy a densely-populated region of radius-flux parameter space (see Figure 4).

\section{CONCLUSIONS}

We report the discovery of four hot Jupiter planets: NGTS-15b, NGTS-16b, NGTS-17b, and NGTS-18b. Each planet was originally identified from photometry from the Next Generation Transit Survey (NGTS), and was confirmed through follow-up observations carried out at the South African Astronomical Observatory (SAAO) with the $1.0 \mathrm{~m}$ and Lesedi telescopes, and radial velocity measurements made with the HARPS, CORALIE and FEROS spectrographs. Additional photometry from the Transiting Exoplanet Survey Satellite (TESS) was also acquired for three of the four targets.

Global fits to these data were produced using the open-source astronomy software package ALLESFITTER (Günther \& Daylan 2020, 2019), and yielded masses, radii, and orbital periods consistent with hot Jupiter planets (see Table 9). Spectral analysis via ISPEC (BlancoCuaresma et al. 2014; Blanco-Cuaresma 2019) and SED fitting via ARIADNE (Vines \& Jenkins 2021) revealed the properties of the host stars in each system, which were all found to be main-sequence G-type stars (see Tables 5, 6, 7, and 8).

As part of our analysis, we noted that all four planets received a level of irradiation that surpassed the expected threshold for the onset of planetary inflation mechanisms (Demory \& Seager 2011; Miller \& Fortney 2011). As such, we sought to characterise any potential inflation by comparing our derived radii with predictions from evolutionary models (Baraffe et al. 2008). In addition, we examined the predicted additional radius change due to inflation, $\Delta R$, through the flux-mass-radius relations outlined in Sestovic et al. (2018). We found that two of the planets are likely inflated when compared with non-inflationary models, and it is reasonable to assume that NGTS-18b, although consistent with both inflated and non-inflated solutions, is also inflated due to its high incident flux. We were unable to draw any firm conclusions on the nature of inflation for NGTS-15b due to the poorly constrained age for this system resulting in a broad range of non-inflated radius values. Furthermore, we note some disparity between the radii derived from global modelling and those predicted by the inflationary forward model of Sestovic et al. (2018), although all four planets were found to fit within the general hot Jupiter population (see Figures $3 \& 4$ ). We suggest that the inclusion of both new hot Jupiter data and additional hyperparameters which describe latent parameters, such as system age, into the Bayesian model may refine the relations further.

\section{ACKNOWLEDGEMENTS}

Based on data collected under the NGTS project at the ESO La Silla Paranal Observatory. The NGTS facility is operated by the consortium institutes with support from the UK Science and Technology Facilities Council (STFC) project ST/M001962/1. RHT is supported by an STFC studentship.

This study is based on observations collected at the European Southern Observatory under ESO programmes 0103.C-0719 and 0104.C-0588 (P.I. Bouchy).

This paper uses observations made at the South African Astronomical Observatory (SAAO).

This paper includes data collected by the TESS mission. Funding for the TESS mission is provided by the NASA Explorer Program. This research has made use of the NASA Exoplanet Archive, which is operated by the California Institute of Technology, under contract with the National Aeronautics and Space Administration under the Exoplanet Exploration Program.

This research has made use of the Exoplanet Orbit Database and the Exoplanet Data Explorer at https://www . exoplanets. org.

This publication makes use of The Data \& Analysis Center for Exoplanets (DACE), which is a facility based at the University of Geneva $(\mathrm{CH})$ dedicated to extrasolar planets data visualisation, exchange and analysis. DACE is a platform of the Swiss National Centre of Competence in Research (NCCR) PlanetS, federating the Swiss expertise in Exoplanet research. The DACE platform is available at https://dace.unige.ch.

This work has made use of data from the European Space Agency (ESA) mission Gaia (https://www. cosmos.esa.int/ gaia), processed by the Gaia Data Processing and Analysis Consortium (DPAC, https://www.cosmos.esa.int/web/ gaia/dpac/consortium). Funding for the DPAC has been provided by national institutions, in particular the institutions participating in the Gaia Multilateral Agreement.

The research leading to these results has received funding from the European Research Council under the European Union's Seventh Framework Programme (FP/2007-2013) / ERC Grant Agreement n. 320964 (WDTracer).

Contributions by authors from the University of Warwick were supported by STFC consolidated grants ST/P000495/1 and ST/T000406/1.

EG gratefully acknowledges support from the David and Claudia Harding Foundation in the form of a Winton Exoplanet Fellowship.

JSJ acknowledges support by FONDECYT grant 1201371, and partial support from CONICYT project Basal AFB-170002.

$\mathrm{ACh}$ and $\mathrm{PhE}$ acknowledge the support of the DFG priority program SPP 1992 "Exploring the Diversity of Extrasolar Planets" (RA 714/13-1)

JIV acknowledges support of CONICYT-PFCHA/Doctorado Nacional-21191829.

MNG acknowledges support from the MIT Kavli Institute as a Juan Carlos Torres Fellow.

We thank the Swiss National Science Foundation (SNSF) and the Geneva University for their continuous support to our planet search programs. This work has been in particular carried out in the 
frame of the National Centre for Competence in Research "PlanetS" supported by SNSF.

\section{DATA AVAILABILITY}

The data underlying this article will be shared on reasonable request to the corresponding author.

\section{REFERENCES}

Allard F., Homeier D., Freytag B., 2012, Philosophical Transactions of the Royal Society of London Series A, 370, 2765

Anderson D. R., et al., 2011, MNRAS, 416, 2108

Asplund M., Grevesse N., Sauval A. J., Scott P., 2009, ARA\&A, 47, 481

Baraffe I., Chabrier G., Barman T., 2008, A\&A, 482, 315

Baranne A., et al., 1996, A\&AS, 119, 373

Blanco-Cuaresma S., 2019, MNRAS, 486, 2075

Blanco-Cuaresma S., Soubiran C., Heiter U., Jofré P., 2014, A\&A, 569, A111

Brahm R., Jordán A., Espinoza N., 2017, PASP, 129, 034002

Castelli F., Kurucz R. L., 2004, A\&A, 419, 725

Charbonneau D., Brown T. M., Latham D. W., Mayor M., 2000, ApJ, 529, L45

Chaushev A., et al., 2019, MNRAS, 488, 5232

Choi J., Dotter A., Conroy C., Cantiello M., Paxton B., Johnson B. D., 2016, ApJ, 823, 102

Costes J. C., et al., 2020, MNRAS, 491, 2834

Demory B.-O., Seager S., 2011, ApJS, 197, 12

Espinoza N., et al., 2016, AJ, 152, 108

Evans D. F., 2018, Research Notes of the American Astronomical Society, 2,20

Foreman-Mackey D., Hogg D. W., Lang D., Goodman J., 2013, PASP, 125, 306

Foreman-Mackey D., Agol E., Ambikasaran S., Angus R., 2017, celerite: Scalable 1D Gaussian Processes in C++, Python, and Julia (ascl:1709.008)

Fortney J. J., Marley M. S., Barnes J. W., 2007, ApJ, 659, 1661

Fressin F., et al., 2013, ApJ, 766, 81

Gaia Collaboration et al., 2016, A\&A, 595, A2

Gaia Collaboration et al., 2018, A\&A, 616, A10

Gray R. O., Corbally C. J., 1994, AJ, 107, 742

Guillot T., Showman A. P., 2002, A\&A, 385, 156

Günther M. N., Daylan T., 2019, Allesfitter: Flexible Star and Exoplanet Inference From Photometry and Radial Velocity, Astrophysics Source Code Library (ascl:1903.003)

Günther M. N., Daylan T., 2020, arXiv e-prints, p. arXiv:2003.14371

Gustafsson B., Edvardsson B., Eriksson K., Jørgensen U. G., Nordlund Å., Plez B., 2008, A\&A, 486, 951

Han E., Wang S. X., Wright J. T., Feng Y. K., Zhao M., Fakhouri O., Brown J. I., Hancock C., 2014, PASP, 126, 827

Hartman J. D., et al., 2012, AJ, 144, 139

Hartman J. D., et al., 2016, AJ, 152, 182

Hauschildt P. H., Allard F., Baron E., 1999, ApJ, 512, 377

Heiter U., et al., 2015, Phys. Scr., 90, 054010

Henden A., Munari U., 2014, Contributions of the Astronomical Observatory Skalnate Pleso, 43, 518

Henry G. W., Marcy G. W., Butler R. P., Vogt S. S., 2000, ApJ, 529, L41

Hsu D. C., Ford E. B., Ragozzine D., Ashby K., 2019, AJ, 158, 109

Husser T. O., Wende-von Berg S., Dreizler S., Homeier D., Reiners A., Barman T., Hauschildt P. H., 2013, A\&A, 553, A6

Jenkins J. S., et al., 2017, MNRAS, 466, 443

Kaufer A., Pasquini L., 1998, in D’Odorico S., ed., Society of PhotoOptical Instrumentation Engineers (SPIE) Conference Series Vol. 3355, Proc. SPIE. pp 844-854, doi:10.1117/12.316798

Kipping D. M., 2013, MNRAS, 435, 2152
Komacek T. D., Thorngren D. P., Lopez E. D., Ginzburg S., 2020, ApJ, 893, 36

Kovács G., Zucker S., Mazeh T., 2002, A\&A, 391, 369

Kurucz R. L., 1993, VizieR Online Data Catalog, p. VI/39

Laughlin G., Crismani M., Adams F. C., 2011, ApJ, 729, L7

Lucy L. B., Sweeney M. A., 1971, AJ, 76, 544

Maldonado J., Villaver E., Eiroa C., Micela G., 2019, A\&A, 624, A94

Maxted P. F. L., 2016, A\&A, 591, A111

Mayor M., Queloz D., 1995, Nature, 378, 355

Mayor M., et al., 2003, The Messenger, 114, 20

Mayor M., et al., 2011, arXiv e-prints, p. arXiv:1109.2497

Miller N., Fortney J. J., 2011, ApJ, 736, L29

Miller N., Fortney J. J., Jackson B., 2009, ApJ, 702, 1413

Queloz D., et al., 2001a, The Messenger, 105, 1

Queloz D., et al., 2001b, A\&A, 379, 279

Raynard L., et al., 2018, MNRAS, 481, 4960

Ricker G., et al., 2014. p. 914320 (arXiv:1406.0151), doi:10.1117/12.2063489

Schlafly E. F., Finkbeiner D. P., 2011, ApJ, 737, 103

Schlegel D. J., Finkbeiner D. P., Davis M., 1998, ApJ, 500, 525

Schönrich R., Binney J., Dehnen W., 2010, MNRAS, 403, 1829

Sestovic M., Demory B.-O., Queloz D., 2018, A\&A, 616, A76

Skrutskie M. F., et al., 2006, AJ, 131, 1163

Southworth J., et al., 2015, MNRAS, 454, 3094

Speagle J. S., 2020, MNRAS, 493, 3132

Spiegel D. S., Burrows A., 2013, ApJ, 772, 76

Stassun K. G., et al., 2019, AJ, 158, 138

Tamuz O., Mazeh T., Zucker S., 2005, MNRAS, 356, 1466

Thorngren D. P., Fortney J. J., 2018, AJ, 155, 214

Thorngren D. P., Fortney J. J., Murray-Clay R. A., Lopez E. D., 2016, ApJ, 831,64

Thorngren D., Fortney J., Lopez E., Burger T., Huber D., 2020, in American Astronomical Society Meeting Abstracts \#236. p. 206.01

Thorngren D. P., Fortney J. J., Lopez E. D., Berger T. A., Huber D., 2021, arXiv e-prints, p. arXiv:2101.05285

Vines J. I., Jenkins J. S., 2021, in prep

Weiss L. M., et al., 2013, ApJ, 768, 14

Wheatley P., et al., 2018, MNRAS, 475, 4476

Wright E. L., et al., 2010, AJ, 140, 1868

Wright J. T., Marcy G. W., Howard A. W., Johnson J. A., Morton T. D., Fischer D. A., 2012, ApJ, 753, 160

\section{APPENDIX A: RADIAL VELOCITY DATA}

This paper has been typeset from a $\mathrm{T}_{\mathrm{E}} \mathrm{X} / \mathrm{L} \mathrm{AT} \mathrm{E} \mathrm{X}$ file prepared by the author. 
Table A1. Summary of radial velocity data from HARPS, CORALIE and FEROS

\begin{tabular}{|c|c|c|c|c|c|c|c|}
\hline Target & $\begin{array}{c}\text { BJD } \\
(-2450000)\end{array}$ & $\begin{array}{c}\mathrm{RV} \\
\left(\mathrm{km} \mathrm{s}^{-1}\right)\end{array}$ & $\begin{array}{c}\mathrm{RV} \text { err } \\
\left(\mathrm{km} \mathrm{s}^{-1}\right)\end{array}$ & $\begin{array}{l}\text { FWHM } \\
\left(\mathrm{km} \mathrm{s}^{-1}\right)\end{array}$ & contrast & $\begin{array}{c}\text { BIS } \\
\left(\mathrm{km} \mathrm{s}^{-1}\right)\end{array}$ & Instrument \\
\hline \multirow{21}{*}{ NGTS-15 } & 8423.852 & 34.764 & 0.102 & 8.204 & 75.167 & 0.421 & CORALIE \\
\hline & 8428.738 & 34.574 & 0.100 & 8.117 & 68.305 & 0.013 & CORALIE \\
\hline & 8493.712 & 34.632 & 0.157 & 8.681 & 89.838 & -0.418 & CORALIE \\
\hline & 8497.732 & 34.561 & 0.123 & 8.252 & 75.461 & 0.146 & CORALIE \\
\hline & 8528.658 & 34.722 & 0.132 & 7.869 & 75.167 & 0.421 & CORALIE \\
\hline & 8538.571 & 34.613 & 0.143 & 8.466 & 72.993 & -1.919 & CORALIE \\
\hline & 8543.582 & 34.545 & 0.137 & 8.398 & 60.515 & 0.073 & CORALIE \\
\hline & 8736.864 & 34.601 & 0.018 & 10.101 & 0.660 & -0.052 & FEROS \\
\hline & 8739.802 & 34.549 & 0.022 & 6.683 & 30.008 & 0.118 & HARPS \\
\hline & 8739.858 & 34.595 & 0.024 & 9.706 & 0.690 & -0.134 & FEROS \\
\hline & 8740.862 & 34.803 & 0.029 & 9.703 & 0.710 & -0.037 & FEROS \\
\hline & 8741.797 & 34.786 & 0.017 & 6.687 & 31.418 & 0.014 & HARPS \\
\hline & 8741.833 & 34.799 & 0.018 & 9.685 & 0.680 & -0.033 & FEROS \\
\hline & 8742.782 & 34.696 & 0.024 & 9.440 & 0.690 & -0.220 & FEROS \\
\hline & 8742.863 & 34.625 & 0.019 & 9.705 & 0.670 & -0.060 & FEROS \\
\hline & 8744.850 & 34.856 & 0.021 & 9.769 & 0.670 & -0.060 & FEROS \\
\hline & 8782.766 & 34.581 & 0.031 & 6.959 & 32.833 & 0.013 & HARPS \\
\hline & 8783.767 & 34.683 & 0.023 & 6.857 & 33.327 & 0.055 & HARPS \\
\hline & 8784.793 & 34.741 & 0.027 & 6.850 & 33.002 & 0.067 & HARPS \\
\hline & 8808.728 & 34.608 & 0.020 & 6.833 & 33.754 & -0.079 & HARPS \\
\hline & 8820.798 & 34.733 & 0.010 & 6.930 & 34.717 & 0.013 & HARPS \\
\hline \multirow{12}{*}{ NGTS-16 } & 8847.635 & 29.126 & 0.014 & 9.970 & 0.600 & 0.063 & FEROS \\
\hline & 8848.650 & 29.078 & 0.016 & 10.217 & 0.600 & -0.075 & FEROS \\
\hline & 8849.653 & 29.079 & 0.015 & 10.058 & 0.590 & 0.035 & FEROS \\
\hline & 8850.616 & 29.209 & 0.014 & 10.002 & 0.600 & -0.012 & FEROS \\
\hline & 8851.619 & 29.219 & 0.014 & 10.034 & 0.600 & -0.003 & FEROS \\
\hline & 8852.614 & 29.072 & 0.015 & 10.112 & 0.600 & -0.063 & FEROS \\
\hline & 8869.629 & 29.155 & 0.009 & 7.650 & 53.996 & -0.026 & HARPS \\
\hline & 8875.604 & 29.190 & 0.009 & 7.697 & 53.738 & -0.026 & HARPS \\
\hline & 8886.579 & 29.136 & 0.011 & 7.693 & 51.044 & -0.040 & HARPS \\
\hline & 8887.572 & 29.058 & 0.010 & 7.649 & 51.040 & -0.032 & HARPS \\
\hline & 8927.511 & 29.148 & 0.009 & 7.625 & 53.670 & -0.039 & HARPS \\
\hline & 8930.509 & 29.090 & 0.030 & 7.605 & 51.292 & -0.003 & HARPS \\
\hline \multirow{20}{*}{ NGTS-17 } & 8737.879 & 34.842 & 0.019 & 10.145 & 0.670 & -0.113 & FEROS \\
\hline & 8739.818 & 34.894 & 0.021 & 10.094 & 0.690 & -0.039 & FEROS \\
\hline & 8741.857 & 34.763 & 0.016 & 9.878 & 0.680 & 0.032 & FEROS \\
\hline & 8742.759 & 34.910 & 0.023 & 10.090 & 0.700 & 0.045 & FEROS \\
\hline & 8744.784 & 34.768 & 0.024 & 10.102 & 0.690 & -0.032 & FEROS \\
\hline & 8745.853 & 35.032 & 0.039 & 10.180 & 0.700 & 0.138 & FEROS \\
\hline & 8757.770 & 34.699 & 0.160 & 8.641 & 46.025 & 0.439 & CORALIE \\
\hline & 8791.689 & 34.813 & 0.147 & 8.823 & 44.152 & 0.130 & CORALIE \\
\hline & 8804.645 & 34.805 & 0.163 & 8.977 & 50.196 & 0.296 & CORLAIE \\
\hline & 8847.672 & 34.801 & 0.017 & 10.239 & 0.660 & -0.037 & FEROS \\
\hline & 8848.674 & 34.702 & 0.017 & 9.955 & 0.660 & 0.051 & FEROS \\
\hline & 8849.677 & 34.945 & 0.019 & 10.190 & 0.660 & -0.024 & FEROS \\
\hline & 8850.692 & 34.925 & 0.016 & 10.165 & 0.670 & -0.016 & FEROS \\
\hline & 8851.662 & 34.742 & 0.016 & 10.145 & 0.670 & -0.019 & FEROS \\
\hline & 8852.669 & 34.769 & 0.015 & 10.187 & 0.670 & -0.030 & FEROS \\
\hline & 8861.678 & 34.573 & 0.176 & 8.926 & 45.527 & -0.231 & CORALIE \\
\hline & 8864.664 & 34.672 & 0.191 & 8.971 & 48.217 & 0.255 & CORALIE \\
\hline & 8869.639 & 34.809 & 0.174 & 8.683 & 46.440 & -0.327 & CORALIE \\
\hline & 8882.644 & 34.981 & 0.184 & 8.650 & 48.722 & 0.467 & CORALIE \\
\hline & 8893.664 & 34.694 & 0.172 & 8.465 & 47.912 & 0.122 & CORALIE \\
\hline
\end{tabular}




\section{Rosanna H. Tilbrook et al.}

Table A2. Summary of radial velocity data from HARPS, CORALIE and FEROS (cont.)

\begin{tabular}{|c|c|c|c|c|c|c|c|}
\hline Target & $\begin{array}{c}\text { BJD } \\
(-2450000)\end{array}$ & $\begin{array}{c}\mathrm{RV} \\
\left(\mathrm{km} \mathrm{s}^{-1}\right)\end{array}$ & $\begin{array}{c}\mathrm{RV} \text { err } \\
\left(\mathrm{km} \mathrm{s}^{-1}\right)\end{array}$ & $\begin{array}{l}\text { FWHM } \\
\left(\mathrm{km} \mathrm{s}^{-1}\right)\end{array}$ & contrast & $\begin{array}{c}\text { BIS } \\
\left(\mathrm{km} \mathrm{s}^{-1}\right)\end{array}$ & Instrument \\
\hline \multirow{17}{*}{ NGTS-18 } & 8624.587 & 5.204 & 0.171 & 8.523 & 54.102 & -0.056 & CORALIE \\
\hline & 8847.770 & 5.207 & 0.017 & 9.177 & 0.620 & 0.034 & FEROS \\
\hline & 8848.777 & 5.151 & 0.018 & 9.912 & 0.650 & 0.067 & FEROS \\
\hline & 8849.800 & 5.171 & 0.019 & 9.321 & 0.630 & -0.067 & FEROS \\
\hline & 8850.817 & 5.340 & 0.018 & 9.872 & 0.650 & 0.056 & FEROS \\
\hline & 8852.793 & 5.207 & 0.016 & 9.746 & 0.650 & 0.053 & FEROS \\
\hline & 8862.770 & 5.173 & 0.160 & 7.402 & 61.367 & -0.494 & CORALIE \\
\hline & 8910.839 & 5.182 & 0.010 & 7.556 & 46.878 & -0.038 & HARPS \\
\hline & 8911.815 & 5.206 & 0.009 & 7.510 & 47.123 & -0.033 & HARPS \\
\hline & 8912.822 & 5.106 & 0.008 & 7.510 & 47.168 & -0.001 & HARPS \\
\hline & 8916.780 & 5.150 & 0.013 & 7.438 & 46.887 & -0.003 & HARPS \\
\hline & 8918.843 & 5.130 & 0.014 & 7.557 & 45.336 & 0.048 & HARPS \\
\hline & 8925.810 & 5.165 & 0.012 & 7.536 & 47.105 & 0.028 & HARPS \\
\hline & 8926.836 & 5.244 & 0.011 & 7.510 & 46.404 & -0.021 & HARPS \\
\hline & 8928.814 & 5.137 & 0.012 & 7.574 & 46.375 & -0.035 & HARPS \\
\hline & 8929.846 & 5.235 & 0.013 & 7.567 & 46.364 & 0.038 & HARPS \\
\hline & 8931.808 & 5.131 & 0.015 & 7.535 & 46.549 & 0.006 & HARPS \\
\hline
\end{tabular}

